Draft version August 8, 2019

Typeset using LATEX twocolumn style in AASTeX63

\title{
On the use of field RR Lyrae as Galactic probes: I. The Oosterhoff dichotomy based on fundamental variables. *
}

\author{
M. Fabrizio,${ }^{1,2}$ G. Bono,${ }^{3,1}$ V.F. Braga,${ }^{4,5}$ D. Magurno, ${ }^{3,1}$ S. Marinoni,${ }^{1,2}$ P.M. Marrese,${ }^{1,2}$ I. Ferraro, ${ }^{1}$ \\ G. Fiorentino, ${ }^{6}$ G. Giuffrida,,${ }^{1}$ G. Innnicola,${ }^{1}$ M. Monelli, ${ }^{7,8}$ G. Altavilla,,${ }^{1,2}$ B. Chaboyer,${ }^{9}$ M. Dall'Ora, ${ }^{10}$ \\ C.K. Gilligan, ${ }^{9}$ A. Layden, ${ }^{11}$ M. Marengo, ${ }^{12}$ M. Nonino, ${ }^{13}$ G.W. Preston,${ }^{14}$ B. Sesar,${ }^{15}$ C. Sneden, ${ }^{16}$ E. Valenti, ${ }^{17}$ \\ F. ThÉvenin, ${ }^{18}$ AND E. ZocCALi ${ }^{4,19}$ \\ ${ }^{1}$ INAF - Osservatorio Astronomico di Roma, Via Frascati 33, 00078, Monte Porzio Catone (Roma), Italy \\ ${ }^{2}$ Space Science Data Center - ASI, Via del Politecnico s.n.c., 00133 Roma, Italy \\ ${ }^{3}$ Department of Physics, Universitá di Roma Tor Vergata, via della Ricerca Scientifica 1, 00133 Roma, Italy \\ ${ }^{4}$ Instituto Milenio de Astrofísica, Santiago, Chile \\ ${ }^{5}$ Departamento de Física, Facultad de Ciencias Exactas, Universidad Andrés Bello, Fernández Concha 700, Las Condes, Santiago, Chile \\ ${ }^{6}$ INAF-Osservatorio di Astrofisica e Scienza dello Spazio di Bologna, Via Piero Gobetti 93/3, I-40129, Bologna, Italy \\ ${ }^{7}$ Instituto de Astrofísica de Canarias, Calle Via Lactea s/n, E-38200 La Laguna, Tenerife, Spain \\ ${ }^{8}$ Departamento de Astrofísica, Universidad de La Laguna, E-38200 La Laguna, Tenerife, Spain \\ ${ }^{9}$ Department of Physics and Astronomy, Dartmouth College, Hanover, NH 03755, USA \\ ${ }^{10}$ INAF-Osservatorio Astronomico di Capodimonte, Salita Moiariello 16, 80131 Napoli, Italy \\ ${ }^{11}$ Bowling Green State University, Bowling Green, OH 43403, USA \\ ${ }^{12}$ Department of Physics and Astronomy, Iowa State University, Ames, IA 50011, USA \\ ${ }^{13}$ INAF-Osservatorio Astronomico di Trieste, Via G.B. Tiepolo, 11, I-34143 Trieste, Italy \\ ${ }^{14}$ Carnegie Observatories, 813 Santa Barbara Street, Pasadena, CA 91101, USA \\ ${ }^{15}$ Deutsche Börse AG, Mergenthalerallee 61, 65760 Eschborn, Germany \\ ${ }^{16}$ Department of Astronomy and McDonald Observatory, The University of Texas, Austin, TX 78712, USA \\ ${ }^{17}$ European Southern Observatory, Karl-Schwarzschild-Str. 2, 85748 Garching bei München, Germany \\ ${ }^{18}$ Université de Nice Sophia-antipolis, CNRS, Observatoire de la Côte d'Azur, Laboratoire Lagrange, BP 4229, F-06304 Nice, France \\ ${ }^{19}$ Pontificia Universidad Católica de Chile, Instituto de Astrofísica, Av. Vicuña Mackenna 4860, 7820436, Macul, Santiago, Chile
}

(Accepted August 6, 2019)

\begin{abstract}
We collected a large data set of field RR Lyrae stars (RRLs) by using catalogues already available in the literature and Gaia DR2. We estimated the iron abundances for a sub-sample of 2,382 fundamental RRLs ( $\Delta$ S method: $\mathrm{Ca}$ II $\mathrm{K}, \mathrm{H} \beta, \mathrm{H} \gamma$ and $\mathrm{H} \delta$ lines) for which are publicly available medium-resolution SDSS-SEGUE spectra. We also included similar estimates available in the literature ending up with the largest and most homogeneous spectroscopic data set ever collected for RRLs $(2,903)$. The metallicity scale was validated by using iron abundances based on high resolution spectra for a fundamental field RRL (V Ind), for which we collected X-shooter spectra covering the entire pulsation cycle. The peak $([\mathrm{Fe} / \mathrm{H}]=-1.59 \pm 0.01)$ and the standard deviation $(\sigma=0.43 \mathrm{dex})$ of the metallicity distribution agree quite well with similar estimates available in the literature. The current measurements disclose a well defined metal-rich tail approaching Solar iron abundance. The spectroscopic sample plotted in the Bailey diagram (period vs luminosity amplitude) shows a steady variation when moving from the metal-poor $([\mathrm{Fe} / \mathrm{H}]=-3.0 /-2.5)$ to the metal-rich $([\mathrm{Fe} / \mathrm{H}]=-0.5 / 0.0)$ regime. The smooth transition in the peak of the period distribution as a function of the metallicity strongly indicates that the longstanding problem of the Oosterhoff dichotomy among Galactic globulars is the consequence of the lack of metal-intermediate clusters hosting RRLs. We also found that the luminosity amplitude, in contrast
\end{abstract}

Corresponding author: Michele Fabrizio

michele.fabrizio@ssdc.asi.it

* Partially based on observations collected under ESO programmes 297.D-5047 (PI. G. Bono) and 083.B-0281 (PI. D. Romano). 
with period, does not show a solid correlation with metallicity. This suggests that period-amplitudemetallicity relations should be cautiously treated.

Keywords: Stars: variables: RR Lyrae — Galaxy: halo — Techniques: spectroscopic

\section{INTRODUCTION}

The advent of space telescopes (HST, Kepler, Gaia) together with long term photometric surveys (OGLEIV, VVV, ASAS, CATILINA) and high-resolution multi-object spectrographs (GIRAFFE@VLT, GMOS@Gemini, AAOmega@AAT) at ground-based 8-10m class telescopes are paving the way to a new golden age for stellar evolution and resolved stellar populations. This means the opportunity to estimate and to measure with unprecedented precision not only intrinsic parameters such as stellar radius, effective temperature and stellar mass (Pietrzyński et al. 2013; Prada Moroni et al. 2012; Marconi et al. 2005), but also the opportunity to constrain the micro (atomic diffusion, opacity, equation of state) and the macro (mixing, rotation, mass loss) physics adopted to construct evolutionary and pulsation models (Salaris 2018).

In spite of this indisputable progress, there are several long-standing astrophysical problems for which, after more than half a century of quantitative astrophysics, we still lack an explanation based on plain physical arguments. The so called Oosterhoff dichotomy is among the most appealing ones. More than seventy years ago, Oosterhoff (1939) recognised that RR Lyraes (RRLs) in Galactic Globular Clusters (GGCs) can be split, according to the mean period of the RRLs pulsating in the fundamental mode (RRab), in two different groups: the Oosterhoff type I $[\mathrm{OoI}]$, with $\left\langle P_{a b}>\sim 0.56\right.$ days, and the Oosterhoff type II [OoII], with longer periods $\left\langle P_{a b}>\sim 0.66\right.$ days. The mean period of the RRLs pulsating in the first overtone (RRc) displays a similar dichotomic distribution with $\left\langle P_{c}\right\rangle \sim 0.31$ days and $\left\langle P_{c}>\sim 0.36\right.$ days in OoI and OoII globulars, respectively. Subsequent spectroscopic investigations enriched the empirical scenario demonstrating that OoI globulars are more metal-rich and cover a broad range in metal abundances, while OoII globulars are more metalpoor stellar systems (Arp 1955; Kinman 1959). Later on, it was also recognised that the population ratio, i.e. the ratio between $R R c$ and the total number of RRLs, is smaller in OoI $\left(\mathrm{N}_{c} / \mathrm{N}_{t o t} \approx 0.29\right)$ than in OoII $\left(\mathrm{N}_{c} / \mathrm{N}_{\text {tot }} \approx 0.44\right)$ globulars (Stobie 1971; Braga et al. 2016; Bono et al. 2016).

The literature concerning the Oosterhoff dichotomy is quite impressive. There is no doubt that Allan Sandage provided in a series of papers covering half century solid empirical evidence concerning the variation of the mean period in field and cluster RRLs (Sandage 1981a,b, 1982, 1990, 1993, 2006, 2010, and references therein). This is the main reason why the same problem is also quoted in the recent literature as the Oosterhoff-ArpSandage period-shift effect (Catelan 2009, and references therein). In this context it is worth mentioning the detailed theoretical investigation provided by Lee et al. (1994) suggesting that a difference in helium content $(\Delta Y=0.03)$ could not explain the observed variation in period, because the predicted variation in period has an opposite sign. The same authors were more in favour of a difference in absolute age of 1-2 Gyr between inner and outer halo globular clusters to take account of the observed variation in period. A difference in luminosity between Oosterhoff I and II groups was also suggested by Lee \& Carney (1999). They investigated RRLs in M2 (OoII) and in M3 (OoI) and found that the former sample was 0.2 magnitude brighter than the latter one. This difference in luminosity was suggested to be caused by a difference in cluster age (Lee et al. 1990). In particular, the RRLs in OoII clusters were considered already evolved off the Zero-Age-Horizontal-Branch (ZAHB), while those in OoI clusters were still near the ZAHB. Moreover, they also suggested, following van den Bergh (1993a,b), there is a difference in kinematic properties between OoI and OoII clusters. Indeed, the former ones appear to have either vanishing or retrograde rotation, while the latter prograde rotation. On the basis of these evidence they suggested that the OoII clusters formed in situ in an earlier epoch, while the OoI clusters either formed later on or accreted. The reader interested in a detailed discussion concerning theoretical and empirical evidence concerning the Oosterhoff dichotomy at the of the last century is referred to the review paper by Caputo et al. (1998).

Evolutionary and pulsation prescriptions were taken into account by Castellani et al. (2003) and they suggested that the difference between OoI and OoII clusters could be explained as a consequence of a difference in the topology of the RRL instability strip (Bono et al. 1995). On the basis of several empirical evidence (the continuity of the mean fundamentalised period, the period distribution in OoI and OoII clusters, the population ratio, the difference between mean fundamental periods and fundamentalised periods) they suggested that the 
so-called OR" region ${ }^{1}$ in OoI clusters is populated by fundamental RRLs, while in OoII clusters is populated by first overtones. The reader interested in a detailed discussion concerning the use of synthetic HB models and their impact on the Oosterhoff dichotomy is referred to Cassisi et al. (2004) and to Catelan (2009).

The possible occurrence of an Oosterhoff III group was also suggested by Pritzl et al. (2003) to take account for the long mean fundamental period of RRLs in two metal-rich clusters (NGC 6388, NGC 6441), but see also Braga et al. (2016). The empirical and theoretical scenario concerning the Oosterhoff dichotomy was further enriched in a recent investigation by Jang \& Lee (2015) in which the authors suggested that the difference among OoI, OoII and OoIII clusters was a consequence of multiple populations in Galactic globulars (Gratton et al. 2004). In particular, they suggested that two/three different star formation episodes with time delays ranging from $\sim 0.5$ to $\sim 1.5 \mathrm{Gyr}$ in inner and outer halo clusters could explain the Oosterhoff-Arp-Sandage period-shift effect.

Large photometric surveys disclosed that Galactic field RRLs display a similar dichotomy in the period distribution (Bono et al. 1997; ASAS: Pojmanski 2002; LONEOS: Miceli et al. 2008; LINEAR: Sesar et al. 2013a). Oddly enough, Local Group galaxies (Draco, Kinemuchi et al. 2008; Ursa Minor, Nemec et al. 1988; Carina, Coppola et al. 2013; Leo I, Stetson et al. 2014) and their globulars (Bono et al. 1994) are characterised by mean fundamental periods that fill the so-called "Oosterhoff gap", i.e. their mean periods range from $\sim 0.58$ to $\sim 0.62$ days (Petroni et al. 2004; Catelan 2009). The lack of Galactic stellar systems with mean periods in the Oosterhoff gap indicates that the environment affects the Oosterhoff dichotomy (Coppola et al. 2015; Fiorentino et al. 2015).

The analysis of this long-standing astrophysical problem was hampered by several empirical biases.

a) - The number of GGCs with a sizeable (more than three dozen) sample of RRLs is limited to 18 out of $\approx 100$ globulars hosting RRLs (Clement et al. 2001). This problem becomes even more severe for Ultra Faint Dwarf galaxies in which the RRL sample never exceeds a dozen (Dall'Ora et al. 2012; Fiorentino et al. 2015).

b) - Although, cluster RRLs have been investigated for more than one century (Bailey 1902), the current samples are far from being complete. This limitation applies to objects centrally located and to low amplitude

\footnotetext{
1 The region of the instability strip in which the RRLs can pulsate either in the fundamental or in the first overtone or in both of them (Bono \& Stellingwerf 1994).
}

variables. The same problem applies to nearby dwarf galaxies due to the lack of a full spatial coverage.

c) - There is mounting empirical evidence that oldand intermediate-age stellar populations in nearby dwarf galaxies display different metallicity distributions (Fabrizio et al. 2015). This means that RRLs in dwarf galaxies might be the progeny of stellar populations characterised by a broader age and/or metallicity distribution (Martínez-Vázquez et al. 2015) when compared with cluster RRLs. The same outcome applies to RRLs in $\omega$ Centauri, the most massive GGC (Braga et al. 2016).

d) - The Bailey diagram (period vs luminosity amplitude) is a solid diagnostic, since it is - together with the period distribution-independent of distance and reddening. To constrain the RRL intrinsic properties, Stetson et al. (2014) and Fiorentino et al. (2015) found that the High Amplitude Short Period (HASP, $P<0.48$ days, $\left.A_{V}>0.75 \mathrm{mag}\right)$ variables are not present in dwarf spheroidals, with the exception of Sagittarius. Detailed investigation among clusters with sizeable sample of RRLs indicate that HASP are only present in systems that are more metal-rich than $[\mathrm{Fe} / \mathrm{H}]=-1.5$ (Monelli et al. 2017).

In the following, we will focus our attention on the pulsation properties of halo RRLs as a function of the chemical composition. The structure of the paper is as follows. In Section 2 we introduce the photometric data sets we adopted to build up the master catalogue of candidate field RRLs. Special attention is given to the cross-match between the RRLs catalogues available in the literature and the Gaia DR2 catalogue. In this section we also mention the criteria we adopted to select candidate Halo RRLs and the approach adopted to identify fundamental and first overtone RRLs. In Section 3 we introduce the spectroscopic data sets we adopted to build up the RRL spectroscopic catalogue. In this section we also describe the approach adopted to retrieve the SDSS-SEGUE medium-resolution spectra and the variant of the $\Delta S$ method adopted to estimate the metallicity of individual RRLs. Moreover, we also discuss the spectroscopic data sets available in the literature. Section 4 deals with the strategy adopted to calibrate and to validate the metallicity scale based on the $\Delta \mathrm{S}$ method. In particular, we focus our attention on V Ind, a fundamental field RRL, for which we have $\mathrm{X}$-shooter spectra covering the entire pulsation cycle. In Section 5 we discuss the metallicity distribution of fundamental RRLs and the comparison with similar estimates available in the literature. Section 6 deals with the fine structure of the Bailey diagram, and in particular, its dependence on the metal content. In this section we also introduce some long-standing open problems 
connected with the Oosterhoff dichotomy and provide new analytical period-metallicity and period-amplitude relations. In Section 7 we briefly discuss the use of the period distribution and of the amplitude distribution to constrain the key properties of the underlying stellar populations. We focus our attention on the RRLs in the Bulge, in Galactic globular clusters, in Magellanic Clouds and in nearby dwarf galaxies. Finally, Section 8 gives a summary of the current results together with a few remarks concerning the future developments of this project.

\section{PHOTOMETRIC DATA SETS}

\subsection{Photometric catalogue}

To provide firm constraints on the metallicity distribution of the Galactic halo we used different photometric and spectroscopic catalogues available in the literature together with the exquisite data provided by the ESA mission Gaia (Gaia Collaboration et al. 2018). A detailed description of the construction of such catalogue is provided in two companion papers (Marinoni et al. 2019, in preparation; Bono et al. 2019, in preparation). Here we briefly summarise the main steps of this process.

We started from the following list of published optical RRL catalogues and surveys: Dambis et al. (2013), CATALINA (Torrealba et al. 2015; Drake et al. 2013a,b, 2014), LINEAR (Sesar et al. 2013a), LONEOS-I (Miceli et al. 2008), NSVS (Hoffman et al. 2009), ROTSE I (Akerlof et al. 2000), QUEST I (Vivas et al. 2004), ASAS (Pojmanski 2002), ASAS-SN (Shappee et al. 2014; Jayasinghe et al. 2018), Magurno et al. (2018).

We build up a single catalogue containing all entries of the quoted literature samples $(\sim 42,000)$, with a particular care to recognise RRLs which were listed in more than one catalogue. We used the algorithm described in Marrese et al. (2019) for sparse catalogues to crossmatch the literature RRLs with Gaia DR2 data, keeping only those stars with a Gaia counterpart. Moreover, we added the new RRLs detected by Gaia (Clementini et al. 2019), which were not included in the literature. The final catalogue includes more than 150,000 candidate RRLs. In order to collect multi-band magnitudes, we used the powerful results of the official Gaia cross-match (Marrese et al. 2017, 2019). In particular, we were able to recover near-infrared (NIR: $J, H, K_{s}$ ) magnitudes from 2MASS PSC (Skrutskie et al. 2006) and VHS DR3 (McMahon et al. 2013), mid-infrared (MIR: $W 1, W 2$ ) magnitudes from allWISE (Wright et al. 2010; Cutri \& et al. 2013) and optical ( $u, g, r, i, z)$ magnitudes from SDSS DR9 (Ahn et al. 2012; Alam et al. 2015). Aiming at a wider wavelength coverage, we also performed, by using the algorithm developed for the large dense sur- veys, the cross-match of Gaia DR2 with ultra-violet catalogue (UV: $F U V, N U V$ ) from GALEX GUVcat_AIS (Bianchi et al. 2017). This means that we build up an $\mathrm{RRL}$ photometric catalogue including magnitudes from the UV to the MIR.

\subsection{Selection of field Halo RRLs}

To improve the selection of field Halo RRLs we applied several selection criteria discussed in the following. It is worth mentioning that they are conservative, i.e. we preferred to possibly lose some candidates, but to avoid spurious contaminations with false identification, and/or blended targets and/or Thin Disk variables such as High Amplitude $\delta$ Scuti. We, also, provided a preliminary estimate of the individual distances by using predicted optical, NIR and MIR Period-Luminosity relations provided by Marconi et al. (2015, 2018). The individual distances were estimated by using apparent MIR and NIR mean magnitudes from allWISE and 2MASS/VHS. Note that in this preliminary step we neglected distances based on Gaia (Bailer-Jones et al. 2018) because the current RRL sample approaches the outermost Halo regions ( 100 kpc). The distances of the RRLs, for which NIR/MIR measurements were not available, were derived by adopting $r, i, z$-band photometry from the SDSS. The distance of the RRLs lacking both MIR/NIR and SDSS photometry was estimated by using the mean $G, G_{\mathrm{BP}}$ and $G_{\mathrm{RP}}$ magnitudes provided by Gaia. The mean of the individual $G$-band measurements was transformed into a mean $R$-band magnitude by using the transformations provided by Evans et al. (2018). Finally, the distances of RRLs, for which at least one of the three quoted Gaia magnitudes was not available, was evaluated by using the canonical visual magnitudemetallicity relation $\left(M_{V}\right.$ vs $\left.[\mathrm{Fe} / \mathrm{H}]\right)$ recently provided by Marconi et al. (2018). For these variables the mean visual magnitude was retrieved from the literature and we adopted a mean Halo metallicity of $[\mathrm{Fe} / \mathrm{H}]=-1.65$ (Layden 1993). The reader interested in a more detailed discussion concerning the Halo metallicity distribution is referred to Sect. 5 . The MIR/NIR and optical apparent mean magnitudes were un-reddened by using the $\mathrm{E}(B-V)$ values from Schlafly \& Finkbeiner (2011), which is the recalibrated extinction map of Schlegel et al. (1998), and the Cardelli et al. (1989) reddening law.

Extended Sources - We removed the objects flagged as "extended" in the 2MASS PSC and allWISE catalogues, by using extKey $(\neq \mathrm{NULL})$ and extFlag $(>1)$ columns respectively.

Position and reddening - In order to avoid the Galactic plane and/or highly reddened areas, we decided to remove the candidate RRLs located either within \pm 2.5 


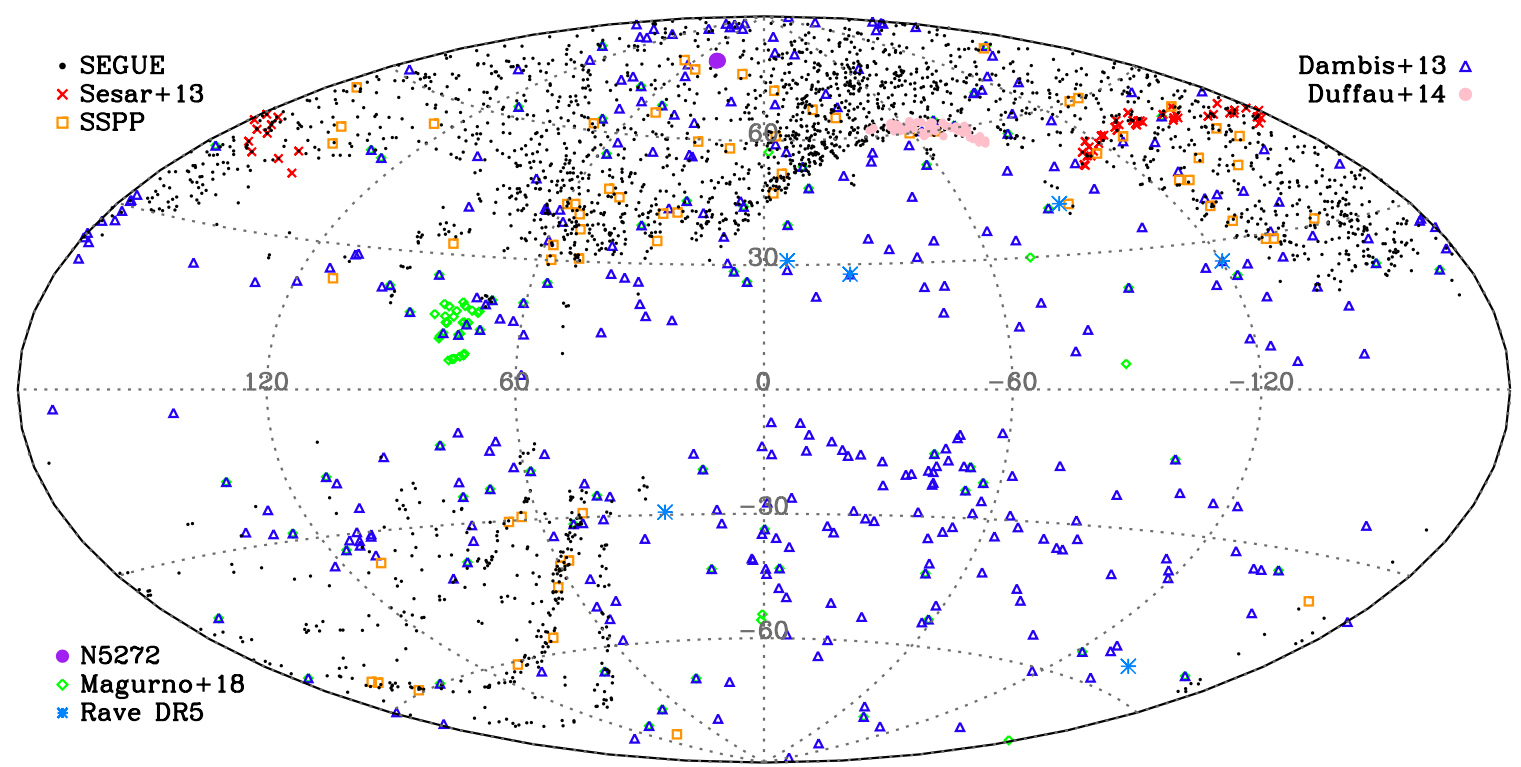

Figure 1. Distribution in Galactic coordinates of the RRL spectroscopic sample (2,903 stars). The black circles show the RRLs with iron abundances based on $\Delta \mathrm{S}$ method on low-resolution SDSS-SEGUE spectra (2,382, SEGUE), while the red crosses display RRLs with iron abundances from Sesar et al. (2013b, 50). Orange squares show RRLs with iron abundances based on SDSS-SSPP indicators (65, SSPP). The blue triangles and the pink circles display RRLs with metallicities from Dambis et al. $(2013,360)$ and Duffau et al. $(2014,57)$. Purple circles are used for the five variables in NGC 5272. Green diamonds show the distribution of the RRLs with iron abundances based on high spectral-resolution spectra (Magurno et al. 2018, 104), while the cyan asterisks refer to iron abundances from Rave DR5 (6).

degrees from the Galactic plane or with a reddening $\mathrm{E}(B-V) \geq 2$ mag.

Spatial overdensities - The distribution of the entire catalogue in Galactic coordinates (X, Y, Z) shows several well-defined overdensities associated either to nearby dwarf galaxies (Magellanic Clouds, Ursa Minor, Draco, Sculptor, Fornax, Carina) or to a globular (NGC 2419) or to the Sagittarius stream (Majewski et al. 2003). They were flagged and the stars belonging to dwarf galaxies or to the globular cluster were removed from the master catalogue. Note that we forced the inclusion of five cluster RRLs belonging to NGC 5272 to increase the sample of spectroscopic standards adopted for calibrating the $\Delta \mathrm{S}$ metallicity scale (see Sect. 3.2).

Spectral Energy Distribution - To further improve the selection of candidate Halo RRLs we also used their Spectral Energy Distribution (SED). The current master catalogue includes multi-band UV (GALEX), optical (SDSS; Gaia; literature: $V, I$ ), NIR (2MASS; VHS) and MIR (allWISE) magnitudes. We took advantage of these independent measurements to estimate several un-reddened mean colours $\left(m_{G}-m_{\lambda}\right)_{0}$ as a function of $\lambda$. On the basis of the RRLs already known in the literature $(\sim 42,000)$ we defined in the colour- $\lambda$ plane a template for the expected RRL colours. We performed an analytical fit of the colour variation and excluded those objects located outside $1 \sigma$ from the analytical fit.
Galactocentric distance - We removed from the sample the candidate RRLs located closer than $4.5 \mathrm{kpc}$ from the Galactic Center. This is a conservative threshold which allows us to neglect bona-fide Galactic Bulge RRLs (Pietrukowicz et al. 2015; Valenti et al. 2018; Zoccali et al. 2018).

After the last selection criterium, we obtained a cleaned master catalogue of candidate Halo RRLs for which, together with the quoted parameters, we also have an estimate of their pulsation period and visual amplitude. For more than $90 \%$ of the sample, we have adopted $\operatorname{Amp}(G)$ from Gaia, while for the remaining $10 \%$, we have adopted literature data from the surveys introduced in Sect.2.1. The light curves of the latter sample were visually inspected and, for a fraction of them, we performed a new estimate of the luminosity amplitudes by using the original time series. The luminosity amplitude in $\operatorname{Amp}(G)$ was transformed into $\operatorname{Amp}(V)$ by using the Eqn.2 from Clementini et al. (2019). In passing we also note that the current luminosity amplitudes are minimally affected by Blazhko modulations, since the cadence and the time interval covered by the adopted long-term photometric surveys cover tens of amplitude modulation cycles. Moreover, to provide a homogenous mode classification we adopted the period-amplitude criterium suggested by Clementini 
et al. (2019):

$$
\frac{2.08-\operatorname{Amp}(G)}{3.5}<P(\text { days })
$$

where $\operatorname{Amp}(G)=[\operatorname{Amp}(V)-0.013] / 1.081 \mathrm{mag}$. Note that we only included RRL candidates with pulsation periods ranging from 0.2 to 1.0 day.

Finally, we neglected both first overtone and mixed mode RRLs by using Eqn. 1 and we ended up with a sample of 44,822 RRab.

\section{SPECTROSCOPY DATA SETS}

The photometric RR Lyrae data sets were complemented with spectroscopic data sets based either on high- or on medium- or on low-resolution spectra. As a whole, we ended up with a sample of 2,903 RRab variables with an iron abundance estimate based on a spectroscopic measurement. Note that in the following we are only dealing with RRab variables, because the spectroscopic calibration adopted for the bulk of the data was devised for this group of variables (see Sect. 3.1.1). The first overtone RRLs will be addressed in a forthcoming paper (Fabrizio et al. 2019, in preparation).

In the following we discuss the different spectroscopic data sets together with the approach adopted to calibrate them on a homogenous metallicity scale. Moreover, we also introduce the approach adopted to validate the spectroscopic diagnostics we are using to estimate iron abundances.

\subsection{SDSS-SEGUE data}

We focussed our attention on the survey "Sloan Extension for Galactic Exploration and Understanding" (SEGUE, Yanny et al. 2009) based on medium resolution $(R \sim 2,000)$ spectra collected with the Sloan Digital Sky Survey DR12 (SDSS, Alam et al. 2015). The photometric and the spectroscopic data collected in this survey are publicly available from the SDSS Science Archive Server (SAS) ${ }^{2}$. The initial step was to download all the available SEGUE spectra for the RRLs in our photometric catalogue. The search was based on the bestObjID from the SpecObjAll table, and we ended up with 2,382 RRab variables for which are available the SEGUE "lite" spectra, i.e. the co-added spectra including up to 38 individual measurements. The sky distribution in Galactic coordinates of the SEGUE sample is shown in Fig. 1 (black symbols).

Fig. 2 shows the un-reddened $G$-band magnitude distribution for different sample of RR Lyrae stars, in particular the SEGUE sample is displayed in panel a) (see

2 https://dr14.sdss.org/home

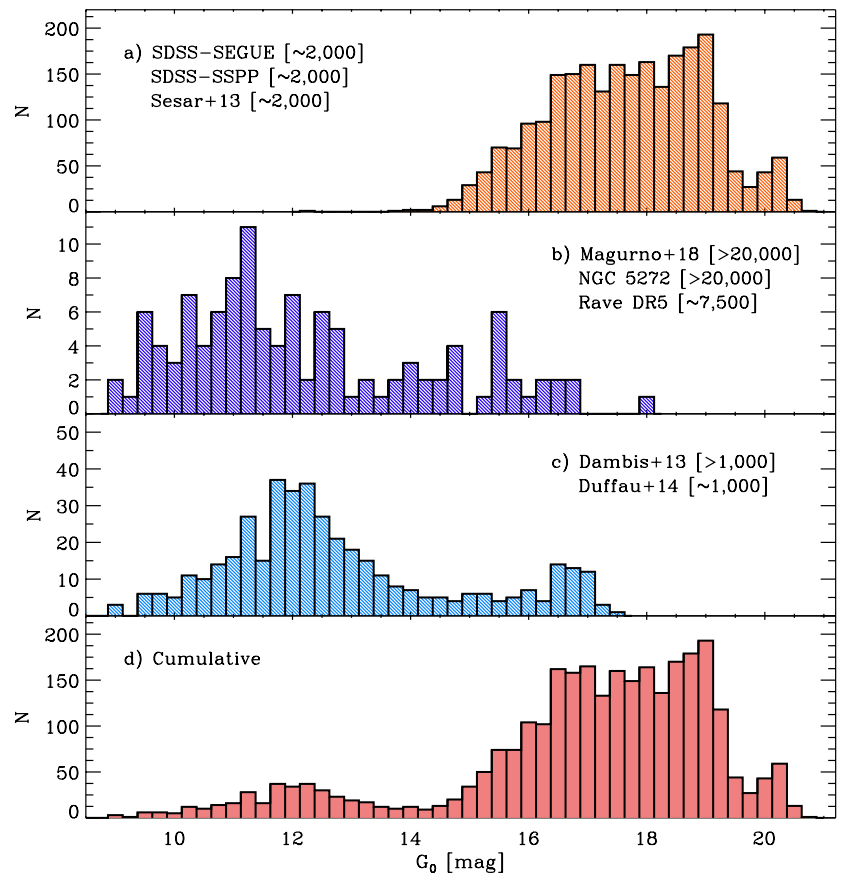

Figure 2. From top to bottom, un-reddened $G$ magnitude distributions of the RRL samples with spectroscopic measurements. The values enclosed in square brackets refer to the spectral resolution of the various samples.

also Sect. 3.2). The individual reddening values were extracted from the Schlegel et al. (1998) dust maps and the updated reddening coefficients from Schlafly \& Finkbeiner (2011), while the extinction in $G$ band was calculated with the Casagrande \& VandenBerg (2018) relation. The key feature of the SEGUE spectra is that they cover a broad spectral range, namely from 3800 to $9200 \AA$ and the majority of the spectra have a mean signal-to-noise ratio larger than $\sim 20$ in the blue region (3900-4900 $\AA$ ). This is the main reason why we decide to use the $\Delta \mathrm{S}$ method introduced half a century ago by G. W. Preston to estimate the iron content of RRLs (see Sect. 3.1.1).

\subsubsection{Metal abundances based on the $\Delta S$ method}

We derived abundances using a variation of the $\Delta \mathrm{S}$ method originally introduced by Preston (1959). In particular, we are following the same approach developed by Layden (1994) which is based on the comparison of pseudo-equivalent width of the Ca II K line, $\mathrm{W}(\mathrm{K})$, and of the mean pseudo-equivalent width of hydrogen lines $\mathrm{H} \delta, \mathrm{H} \gamma$ and $\mathrm{H} \beta, \mathrm{W}(\mathrm{H})$. The pseudo-equivalent widths (hereafter, EW) were measured on SEGUE spectra by 

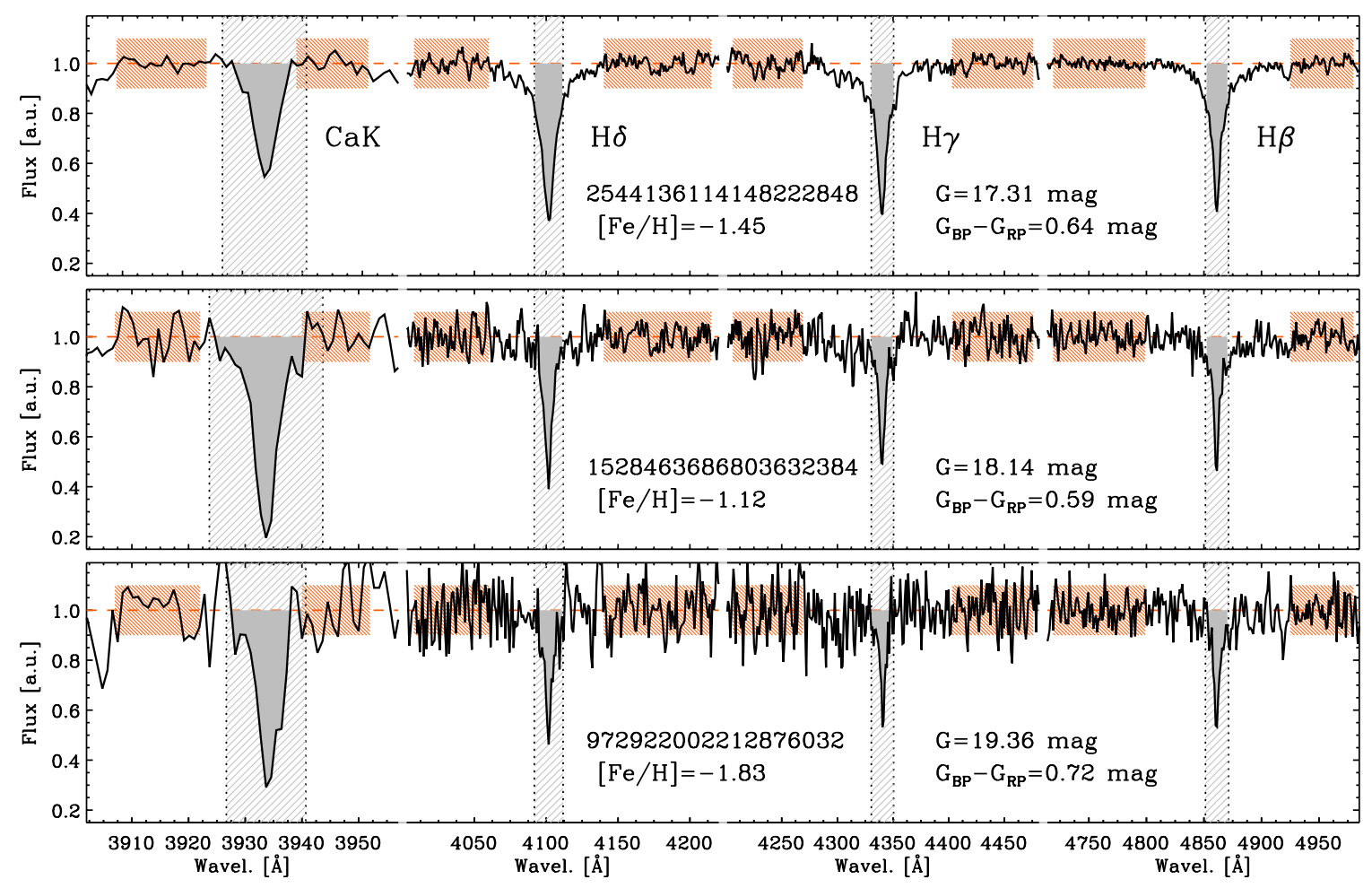

Figure 3. Normalised SEGUE spectra for three field RR Lyrae. The hatched orange regions outline the wavelength range used to estimate the continuum mean flux (red dashed line), while the hatched light grey regions and the dotted vertical lines display the wavelength interval in which the equivalent width is measured. The dark grey regions display the EWs for the four spectroscopic diagnostics: $\mathrm{CaII} \mathrm{K}, \mathrm{H} \delta, \mathrm{H} \gamma$ and $\mathrm{H} \beta$.

using an IDL $^{3}$ version of the original EWIMH program ${ }^{4}$ written by one of us (A. Layden). The algorithm defines, for each spectral feature, a pseudo-continuum level as a straight line (dashed red line in Fig. 3) between the mean intensity and the mean wavelength points of two continuum bands (see table 5 in Layden 1994 and the orange hatched areas in Fig. 3).

The EW (dark grey area showed in Fig. 3) is defined as the area enclosed by the limits in wavelength of the specific spectral feature (vertical light grey hatched area) and the pseudo-continuum of the spectrum. This area is then divided by the mean height of the continuum inside the specific spectral feature. The three panels of Fig. 3 display the details of the measurements for three targets with different magnitudes, colours and metallicities (see labeled values).

A crucial issue in the use of the $\Delta \mathrm{S}$ method is the calibration of the measured EWs onto a "standard sample" of EWs. The list of the 17 standard stars is given in Table 6 of Layden (1994). Unfortunately, there is no overlap between the SEGUE survey and the set of

\footnotetext{
3 https://www.harrisgeospatial.com/Software-Technology/IDL

${ }^{4}$ http://physics.bgsu.edu/ layden/ASTRO/DATA/EXPORT/ EWIMH/ewimh.htm
}

spectroscopic "standards" adopted by Layden. This means that we cannot directly use the relations defined by Layden (1994) to derive the iron abundance. Fortunately enough, in a recent investigation, one of us (Sesar et al. 2013b) provided an independent calibration of the $\Delta \mathrm{S}$ method to investigate the metallicity distribution of RRLs in the Orphan Stream. They collected low-resolution spectra ( $\mathrm{R} \sim 1350)$ with the Double Spectrograph (DBSP, Oke \& Gunn 1982) available at the Palomar $5.1 \mathrm{~m}$ telescope for 50 Orphan Stream RRLs. Moreover, they also observed eight out of the 17 standard stars and provided four linear relations between the EWs measured on DBSP spectra and those based on the Layden's spectroscopic standards (see equations 9-12 in Sesar et al. 2013b).

The Orphan Stream spectroscopic data set and the SEGUE data set have 27 RRLs in common. Among them, ten have a sufficient signal-to-noise ratio to calibrate the EWs measured on SEGUE spectra onto the EWs measured on DBSP spectra. More specifically, we measured the EWs on both DBSP and on SEGUE spectra (degraded to the DBSP spectral resolution). The individual measurements concerning the $\mathrm{Ca}$ II $\mathrm{K}$ line and the three hydrogen lines are plotted in Fig. 4 and show, within the errors, a linear trend over a broad range of 

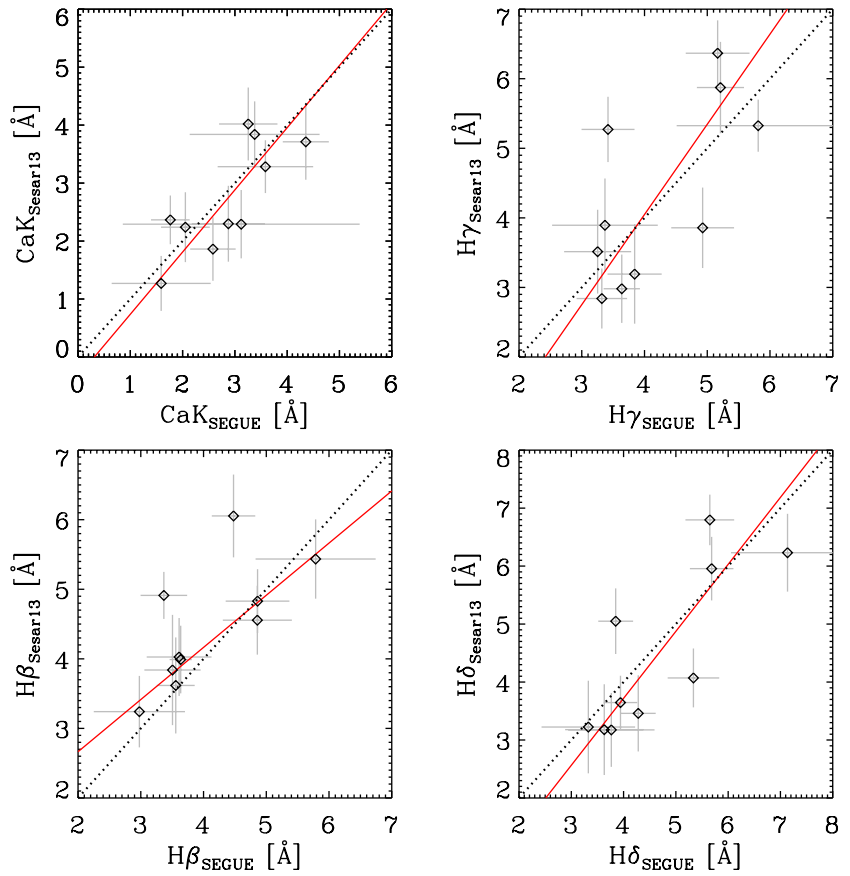

Figure 4. Comparison between the equivalent widths measured on the Sesar et al. (2013b) spectra and those based on the SEGUE spectra degraded to the DBSP resolution ( $\mathrm{R} \sim 1300)$ for the ten RRLs in common. The dotted line shows the bisector of the plane. The linear fits used to transform the EWs based on SEGUE spectra into the Sesar's equivalent width system is plotted as a red line.

EWs. We also performed four linear fits to transform the current EW measurements into the EW system defined by Sesar et al. (2013b, see red lines). The linear relations are the following:

$$
\begin{aligned}
\mathrm{CaK}_{\mathrm{Sesar} 13} & =1.07 \cdot \mathrm{CaK} \mathrm{K}_{\mathrm{SEGUE}}-0.34 \\
\mathrm{H} \beta_{\text {Sesar13 }} & =0.75 \cdot \mathrm{H} \beta_{\mathrm{SEGUE}}+1.17 \\
\mathrm{H} \gamma_{\text {Sesar13 }} & =1.30 \cdot \mathrm{H} \gamma_{\mathrm{SEGUE}}-1.14 \\
\mathrm{H} \delta_{\text {Sesar13 }} & =1.16 \cdot \mathrm{H} \delta_{\mathrm{SEGUE}}-0.90
\end{aligned}
$$

Finally, the EWs in the system defined by Sesar et al. (2013b) were transformed into the system defined by Layden (1994) standard stars. Moreover, following Layden (1994), the Ca II K EWs were also corrected for interstellar Ca II absorption using the Beers (1990) model:

$$
W\left(K_{0}\right)=W(K)-W_{\max }\left(1-e^{-|z| / h}\right) / \sin |b|,
$$

where $W_{\max }=0.192 \AA, h=1.081 \mathrm{kpc}, b$ is the Galactic latitude, and $z$ is the height above the Galactic plane in kpc.

The iron abundances were evaluated by inverting equation 7 of Layden (1994):

$$
[\mathrm{Fe} / \mathrm{H}]=\frac{W\left(K_{0}\right)-a-b W(H)}{c+d W(H)},
$$
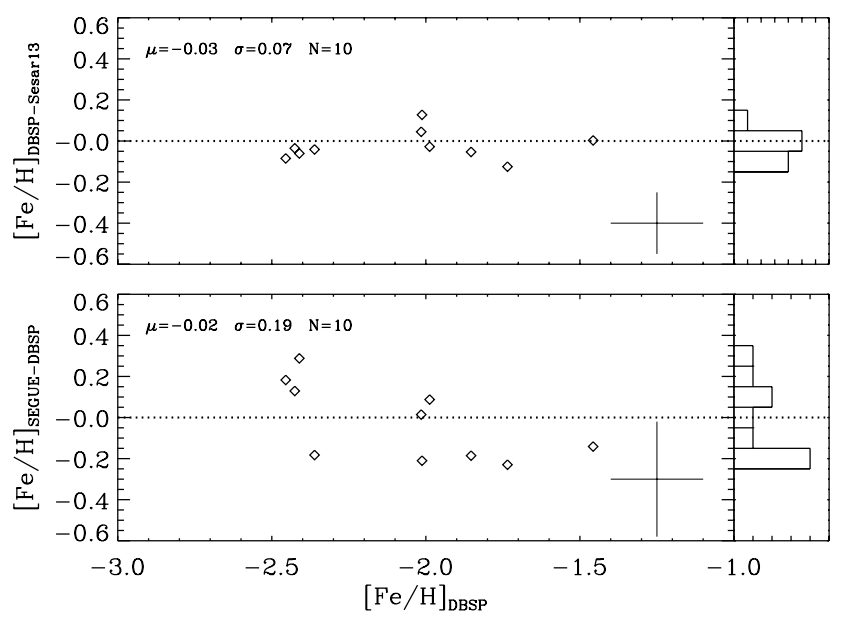

Figure 5. Top: Difference between the iron abundances estimated by us on the DBSP spectra and those provided by Sesar et al. (2013b). Bottom: Difference between the iron abundances based on the $\Delta S$ method applied to the rebinned SEGUE spectra and the iron abundance we estimated with the $\Delta \mathrm{S}$ method applied to Sesar et al. (2013b) DBSP spectra, for ten stars in common. The mean and the standard deviation of the differences are also labelled.

where $a=13.858, b=-1.185, c=4.228$ and $d=-0.32$. To validate the current approach, we compared our iron abundances with those provided by Sesar et al. (2013b). The top panel of Fig. 5 shows the comparison between the $[\mathrm{Fe} / \mathrm{H}]$ measured by us on the DBSP spectra and the $[\mathrm{Fe} / \mathrm{H}]$ abundances measured by Sesar et al. (2013b). We found a very good agreement, and indeed, the mean difference is minimal $(-0.03 \mathrm{dex})$ and the dispersion is negligible $(0.07 \mathrm{dex})$. The bottom panel of the same Fig. 5 shows a similar comparison, but between the $[\mathrm{Fe} / \mathrm{H}]$ abundances based on SEGUE and on DBSP spectra. We found again a good agreement between the two data sets, and indeed the mean difference is minimal $(-0.02 \mathrm{dex})$ and the dispersion is smaller than $0.2 \mathrm{dex}$. These results further support the approach we devised to calibrate the $[\mathrm{Fe} / \mathrm{H}]$ abundance onto those provided by Sesar et al. (2013b), and subsequently onto the Layden's metallicity scale (Layden 1994), which, in turn, is rooted onto the Zinn \& West (1984) globular cluster metallicity scale.

\subsection{Spectroscopic data sets available in literature}

In order to validate and to enlarge the SDSS-SEGUE dataset, we also included the large sample of iron abundances collected by Magurno et al. (2018, Tab.10) and based on high-resolution spectra $(\mathrm{R}>20,000)$. The whole dataset was scaled to the Asplund et al. (2009) Solar reference. The entire sample includes 134 objects, but we only took into account fundamental RRLs (104). 
Note that this sample mainly includes bright nearby RRLs, and indeed the limiting magnitude is $G \sim 17$ mag.

To increase the spatial distribution and the size of high-resolution sample, the quoted data set was complemented with the iron abundances retrieved from the Radial Velocity Experiment DR5 (RAVE, Kunder et al. 2017; Casey et al. 2017). The iron abundances for six RRLs are based on spectra covering the Ca-triplet region $(8410-8795 \AA)$ with a spectral resolution $\mathrm{R} \sim 7,500$. Furthermore, the sample was complemented with five cluster RRLs belonging to NGC 5272. We adopted the RRLs listed in Clement et al. (2001), and the cluster iron abundances provided by Harris (2010). The three data sets defining the high-resolution (HR) sample were scaled to the same Asplund et al. (2009) Solar reference. Their $G_{0}$-band magnitude distribution is shown in the panel b) of Fig. 2.

The literature sample was also complemented with the iron abundances collected by Dambis et al. (2013), based on a mix of low-, medium- and high-resolution spectra. This data set includes 402 RRLs and among them 360 were included in the current spectroscopic catalogue. The bulk of this data set comes either from the $\Delta \mathrm{S}$ measurements provided by Layden (1994), by Fernley et al. (1998) and by Kinman et al. (2007). Panel c) of Fig. 2 shows the magnitude distribution of this data set.

Furthermore, we complemented the literature sample by including the metallicities of RRLs, based on $\Delta S$ method, identified by the QUEST survey and published by Duffau et al. (2014). This data set is based on a mix of low- and medium-resolution spectra. This sample includes 82 RRLs and among them 57 are RRab variables belong to the current spectroscopic catalogue. Its magnitude distribution is shown in the panel c) of Fig. 2, mainly defining the tail between $G_{0} \sim 16$ and $18 \mathrm{mag}$.

Moreover, the SEGUE survey also provides an independent estimate of the iron abundance by using their Stellar Parameter Pipeline (SSPP, Lee et al. 2008a). The SSPP uses multiple techniques to measure the radial velocities, to estimate the fundamental stellar parameters (effective temperature, surface gravity) and to determine the iron abundance (Lee et al. 2008b; Allende Prieto et al. 2008). In this context, it is worth mentioning that the iron abundances provided by SSPP are based on twelve independent spectroscopic diagnostics. The pipeline gives a mean best value (FEHADOP) together with its uncertainty. These iron estimates define the SDSS-SSPP sample and among them 65 were included in the current spectroscopic catalogue. The cumulative magnitude distribution of the entire spectroscopic catalogue is shown in panel d) of Fig. 2.

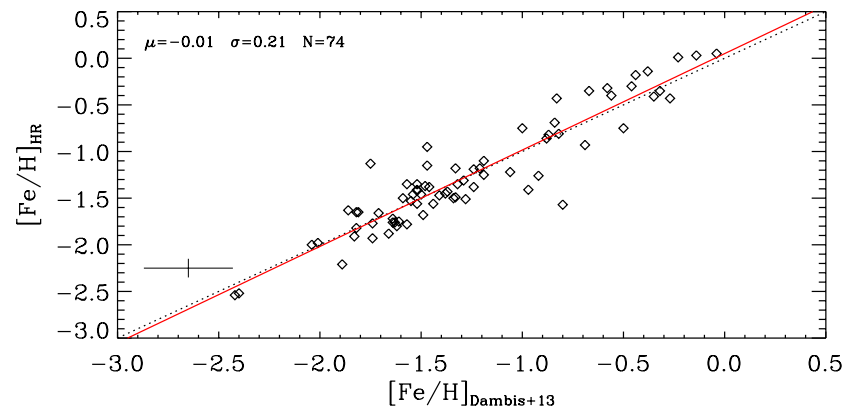

Figure 6. Calibration of Dambis et al. (2013) iron abundances with the iron abundances based on high-resolution spectra collected by Magurno et al. (2018). The linear fit used to transform Dambis et al. (2013) iron abundances into the HR sample is plotted as a red line. The dotted line shows the bisector of the plane

\section{CALIBRATION AND VALIDATION OF THE SPECTROSCOPIC DATA SET}

\subsection{Spectroscopic Calibration}

To provide a homogenous metallicity scale for the different spectroscopic data sets discussed in the previous section we took into account stars in common between the HR sample (pivot sample), the medium- and lowresolution data sets. We found 74 RRLs in common between the HR sample and the Dambis et al. (2013) sample. Data plotted in Fig. 6 show that the two data sets agree quite well, and indeed, the mean difference is minimal $(-0.01 \mathrm{dex})$, while the standard deviation is 0.21 dex. The dispersion is mainly a consequence of the intrinsic errors of the two data sets (see error bars in the bottom left corner). Note that the 74 RRLs in common cover a wide range in $[\mathrm{Fe} / \mathrm{H}]$ abundances (more than 2 dex) and we found evidence of a mild drift when moving from the metal-poor to the metal-rich regime. We performed a linear regression and we found the following linear relation:

$$
[\mathrm{Fe} / \mathrm{H}]_{\mathrm{HR}}=0.05+1.03 \cdot[\mathrm{Fe} / \mathrm{H}]_{\text {Dambis }+13}
$$

to move the Dambis iron abundances into the HR metallicity scale.

The HR sample was joined with the Dambis sample, defining a new data set of 401 RRLs as the "calibration sample". The iron abundances of the calibration sample were compared with values based on the $\Delta \mathrm{S}$ method we applied to the SEGUE spectra. The number of RRLs in common is eleven and the top panel of Fig. 7 shows the comparison. We found a systematic offset of $-0.26 \mathrm{dex}$ (with a standard deviation of $0.29 \mathrm{dex}$ ) and it was applied to the iron abundances based on the $\Delta \mathrm{S}$ method.

The middle panel of Fig. 7 shows the difference of $[\mathrm{Fe} / \mathrm{H}]$ based on $\Delta \mathrm{S}$ method between the SEGUE and 

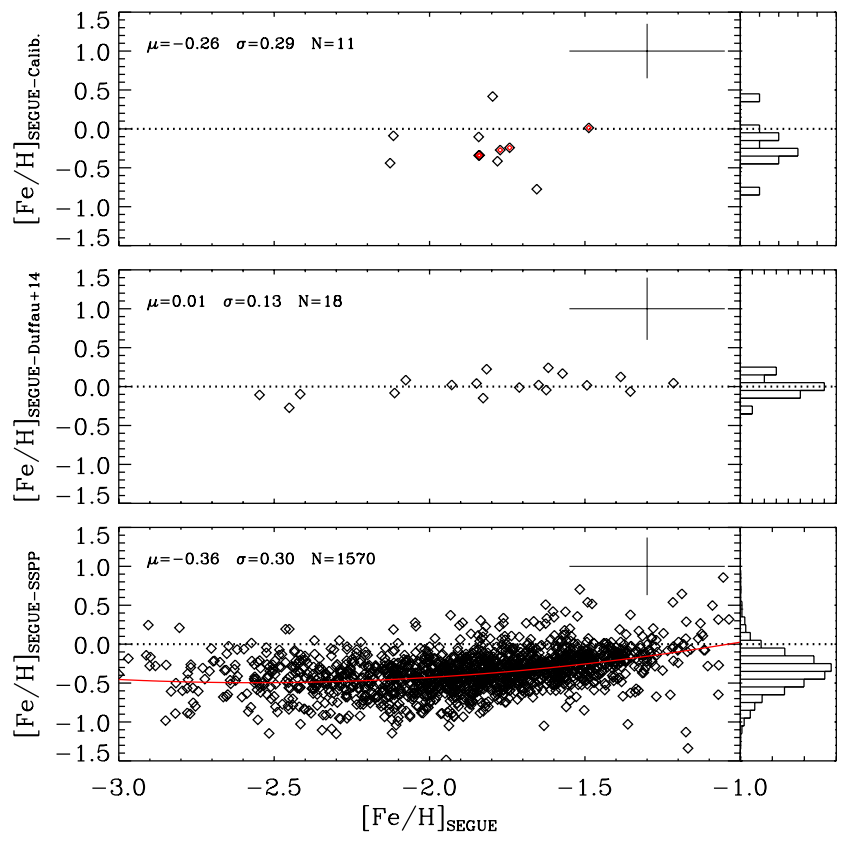

Figure 7. Top: Comparison between the iron abundances based on the current $\Delta S$ method and those based on the calibration sample (see Sect. 4.1). The red diamonds mark the Globular Cluster RRLs. Middle: Comparison between the iron abundances based on the current $\Delta \mathrm{S}$ method and those based on the Duffau et al. (2014) sample. Bottom: Comparison between the iron abundances based on the $\Delta \mathrm{S}$ method and those based on the SDSS-SSPP method. The quadratic relation to transform the SDSS-SSPP into the current metallicity scale is also plotted as a red line. The mean and the standard deviation of the difference are also labelled together with the sample size.

Duffau et al. (2014) samples. The two samples have 18 RRab variables in common and the mean difference in metallicity is vanishing ( $0.01 \mathrm{dex})$ with a dispersion of 0.13 dex. This result allows us to treat the Duffau et al. (2014) sample as the SEGUE one, by applying the same metallicity scale.

Finally, we calibrated the iron abundances based on the SDSS-SSPP metallicity determinations into the same HR metallicity scale. The number of RRLs in common among the HR sample plus our $\Delta \mathrm{S}$ sample and SDSS-SSPP is larger than 1,500 objects. Data plotted in the bottom panel of Fig. 7 shows that the mean difference is -0.36 dex. Note that the current finding agrees with a similar result (difference equal to $-0.36 \mathrm{dex}$ ) obtained by Sesar et al. (2013b) by using an independent spectroscopic data set. The dispersion we found is 0.30 dex and it is fully supported by the intrinsic errors of the different samples (see the error bars in the top right corner).

We also found that a quadratic relation allows us to calibrate the SDSS-SSPP iron abundances into the HR metallicity scale:

$$
\begin{array}{r}
\text { FEHADOP }^{*}=-0.65+0.60 \cdot(\text { FEHADOP }+0.26) \\
-0.05 \cdot(\text { FEHADOP }+0.26)^{2}
\end{array}
$$

Once this relation was applied to the SSPP iron abundances we obtained a null residual with a dispersion of 0.27 dex.

\subsection{Validation of the spectroscopic measurements: individual vs co-added spectra}

We have already mentioned in Sect.3.1, that the metallicity estimates rely on the application of the $\Delta S$ method to the SDSS-SEGUE co-added spectra. The coadded spectrum is typically based on three back-to-back 900s individual spectra collected but, in order to achieve highest signal-to-noise ratio, the individual spectra can also spread over days (Bickerton et al. 2012). Moreover, it is worth mentioning that the spectra were collected at random pulsation phases. To quantify the impact that the co-adding of spectra collected at random pulsation phases have on the metallicity estimates, we evaluated the difference between the metallicity estimates based on the co-added spectrum with the metallicity based on the application of the $\Delta \mathrm{S}$ method on the individual spectra. To validate the approach, we selected the individual spectra with a signal-to-noise ratio larger than $\sim 20$. We ended up with a sample of more than 1,000 RRab variables.

Figure 8 shows the distribution of the difference between the mean of individual $[\mathrm{Fe} / \mathrm{H}]$ estimates and the $[\mathrm{Fe} / \mathrm{H}]$ measured on the co-added spectrum. The Gaussian fit to the distribution (blue curve) gives a peak of $\sigma=0.06$ dex, while the standard deviation of the measurements is 0.16 dex, i.e. a factor of two smaller than the standard deviation of the calibration of the

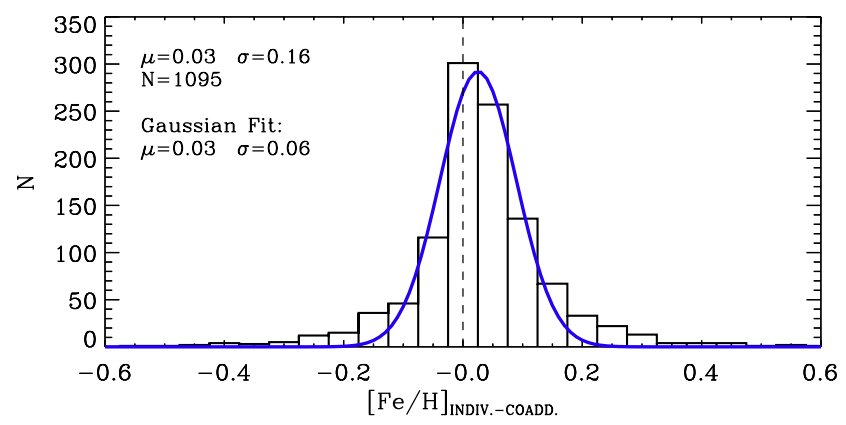

Figure 8. Distribution of the difference between the mean of the individual metallicity measurements and the metallicity measured on the co-added spectrum for a sample of 1,095 RRLs. The blue curve shows the Gaussian fit to the distribution. The mean and the standard deviation of the sample and of the Gaussian fit are also labelled. 
$\Delta \mathrm{S}$ method with the HR metallicity scale (0.29 dex, see also Sect.5). Moreover and even more importantly, the current evaluation agrees quite well with similar similar estimated provided by Drake et al. (2013a, 0.22 dex) by using SDSS spectra. In passing we note that the modest value in the mean difference further supports the use the co-added spectra, typically characterised by higher signal-to-noise ratios, to determine metal abundances of $\mathrm{RR}$ Lyrae by using the $\Delta \mathrm{S}$ method.

\subsection{Validation of the spectroscopic measurements: $V$ Ind}

It is worth noting that the SDSS-SEGUE spectroscopic data were collected at random phases along the pulsation cycle, with exposure times of 15 min (Smee et al. 2013). This means that a fraction of the spectra could have been collected along the rising branch. The rising branch has always been avoided in the spectroscopic analysis and in the application of the BaadeWesselink method (Storm et al. 1994). The reasons are manifold. Dating back to more than half century ago, Preston \& Paczynski (1964) demonstrated on empirical basis that across these phases a strong shock is formed and propagates towards the outermost regions. This causes the occurrence of line doubling and P Cygni profile, further supporting the presence of strong nonlinear phenomena in the outermost layers. This empirical scenario was soundly supported by nonlinear, convective models taking account for time dependent convective transport suggesting that the efficiency of the convective transport attains its maximum efficiency along the rising branch. This is not a severe limitation, since the time interval between minimum and maximum light is of the order of $10 \%$ of the pulsation cycle. However, these are the reasons why the $\Delta S$ method was not applied to this portion of the pulsation cycle (Freeman \& Rodgers 1975; Layden 1993). In dealing with large spectroscopic samples we cannot exclude that a minor fraction can also be collected during these pulsation phases. Moreover, we still lack quantitative constraints of the impact that these phenomena have on abundance estimates based on the $\Delta \mathrm{S}$ method.

In a recent investigation, Magurno (2018) estimated iron abundance of a field, short-period ( $P \sim 0.48$ day), large-amplitude $\left(\mathrm{A}_{V} \sim 1.07 \mathrm{mag}\right.$, Monson et al. 2017) fundamental RRL: V Ind. He adopted twelve mediumresolution ( $\mathrm{R} \sim 10,000-18,000)$, high signal-to-noise ratio $(\sim 200)$ spectra collected with X-shooter (Vernet et al. 2011) at ESO/VLT ${ }^{5}$. The key advantage of X-shooter is

${ }^{5}$ Based on observations collected under ESO programme ID 297.D5047(A), PI. G. Bono.

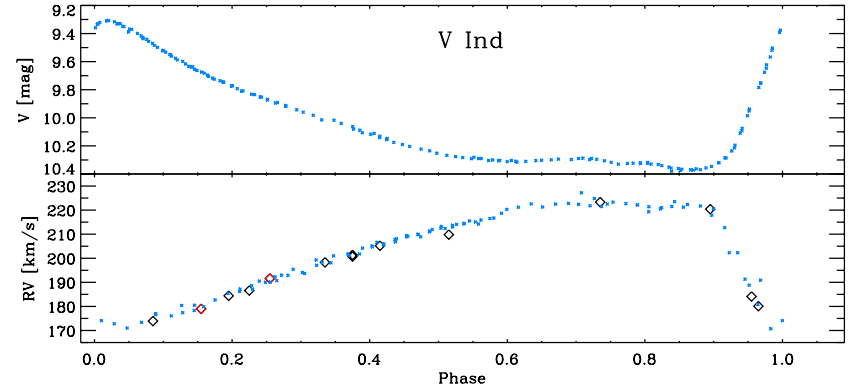

Figure 9. Visual light curve of V Ind (top panel) and radial velocity curve (bottom panel) as function of the pulsation phase (blue crosses Clementini et al. 1990). Black diamonds mark the radial velocities based on X-shooter spectra (Magurno 2018), while the red ones are used for UVES ones (Pancino et al. 2015).

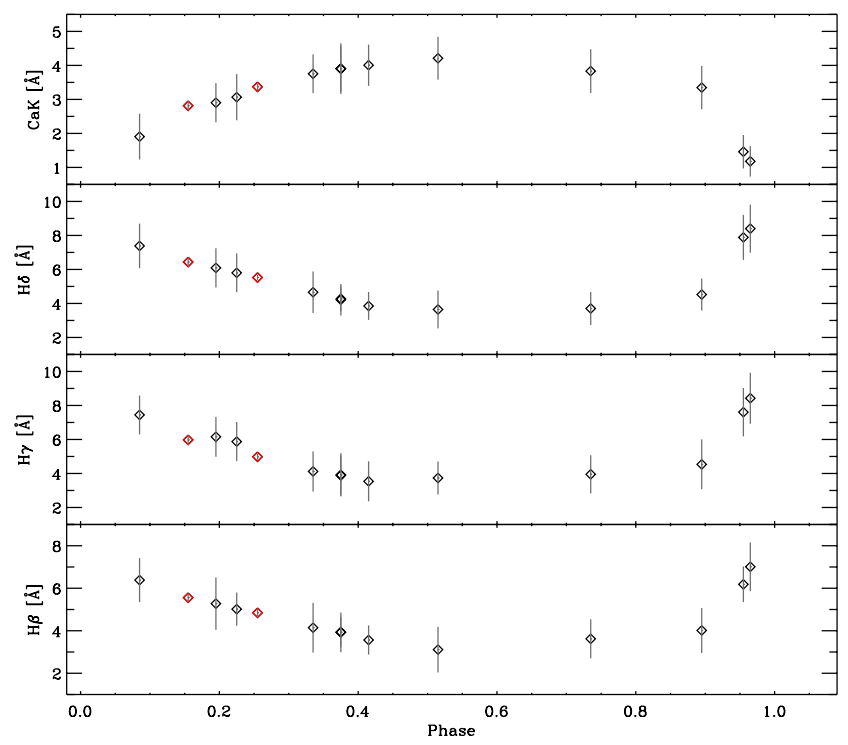

Figure 10. Equivalent widths of the four spectroscopic diagnostics adopted to apply the $\Delta S$ method to fundamental RRL V Ind as a function of the pulsation phase. The symbols are the same as in Fig. 9.

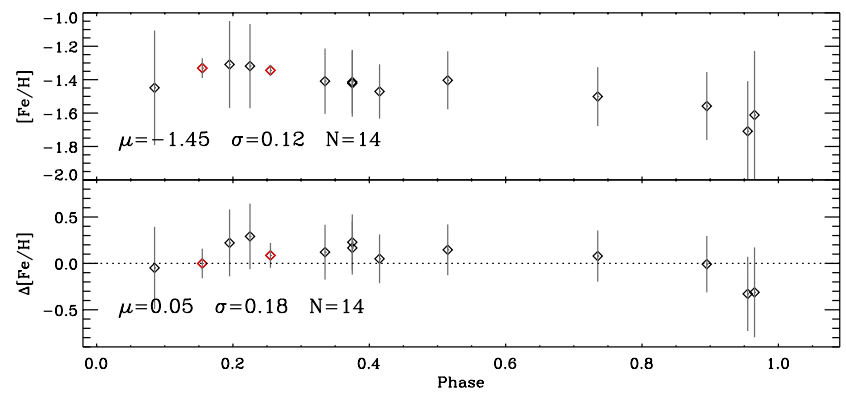

Figure 11. Top: iron abundances for V Ind based on the $\Delta \mathrm{S}$ method. Bottom: difference in iron abundance with the iron values provided by Magurno (2018) and by Pancino et al. (2015). The symbols are the same as in Fig. 9. 
the possibility to simultaneously cover a very wide wavelength regime, ranging from $\sim 3000$ to $\sim 25,000 \AA$. Moreover, the spectra cover the entire pulsation cycle and the exposure times are quite short (120-180 sec). The target is quite bright ( $V=9.97 \mathrm{mag}$ ) for an $8 \mathrm{~m}$ class telescope and we decided to use a narrow slit of $0.4^{\prime \prime}$, obtaining a spectral resolution in the optical range of the order of 18,000 . It is worth mentioning that $\mathrm{V}$ Ind is an acid test to investigate the metallicity estimates along the pulsation cycle, since it is among the RRLs with the largest pulsation amplitudes. On the basis of these spectra, Magurno (2018) measured the iron abundance of $\mathrm{V}$ Ind as function of its pulsation phase. Fig. 9 shows the visual light curve (top) and the radial velocity (bottom) as function of the pulsation phase (blue crosses from Clementini et al. 1990). The radial velocity measurements based on X-shooter spectra are marked with black diamonds. To improve the sampling along the pulsation cycle, we also included two high-resolution ( $\mathrm{R} \sim 40,000)$ spectra collected with UVES (Dekker et al.

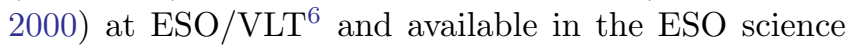
archive (red diamonds in Fig. 9). Fortunately enough, both the X-shooter and the UVES spectra cover the wavelength range from Ca II $\mathrm{K}$ to $\mathrm{H} \beta$ lines.

To validate the adopted $\Delta \mathrm{S}$ method as function of the pulsation phase, the quoted high-resolution spectra were degraded and re-binned to the spectral resolution $(\mathrm{R} \sim 2000)$ and sampling $(\Delta \log \lambda=0.0001)$ of SDSSSEGUE spectra. The quality of the re-binned spectra was quite good, with a signal-to-noise ratio of $\sim 200$ and they appear to be quite similar to the best SEGUE spectra (see Fig. 3). We applied the $\Delta \mathrm{S}$ method described in Sect. 3.1.1 to the re-binned spectra and the same spectroscopic calibration described in Sect. 4.1 to transform the EWs into iron abundance. Fig. 10 shows the EW measurements of the spectral features involved in the $\Delta \mathrm{S}$ method and they show the expected trend in Ca II K and $\mathrm{H}$ lines. Data plotted in the top panel of this figure clearly show that the EW of the Ca II K line steadily increases when moving along the decreasing branch, it attains its maximum across the phases of minimum light and it starts to decrease along the rising branch. The trend for the $\mathrm{H}$ lines is exactly a mirror image of the Ca II K line. This means that the ratio between the EWs of $\mathrm{Ca} I \mathrm{~K}$ and $\mathrm{H}$ lines remains almost constant over the entire cycle. This is the reason why the $[\mathrm{Fe} / \mathrm{H}]$ abundances based on $\Delta \mathrm{S}$ method do not show a phase de-

${ }^{6}$ Based on observations collected under ESO programme ID 083.B0281(A), PI. D. Romano. pendence. Indeed, the iron abundances attain similar values, within the errors, over the entire pulsation cycle.

The top panel of Fig. 11 shows the $[\mathrm{Fe} / \mathrm{H}]$ estimates as a function of pulsation period: the mean value is -1.45 dex, while the standard deviation of the measurements is 0.12 dex. The current mean iron abundances agree quite well similar estimates provided by Magurno (2018) and by Pancino et al. (2015). In fact, the bottom panel of Fig. 11 shows the phase-to-phase difference in iron abundance based on $\Delta \mathrm{S}$ compared to those from Magurno (2018) and Pancino et al. (2015) for Xshooter and UVES data, respectively. The comparison indicates that iron abundances based on $\Delta \mathrm{S}$ method and those based on high-resolution spectra attain similar values, indeed the mean is vanishing (0.05 dex), while the standard deviation is 0.18 dex. More important, the $[\mathrm{Fe} / \mathrm{H}]$ values agree within the errors also along the raising branch of $\mathrm{V}$ Ind. Finally, the quoted results allow us to use the derived $[\mathrm{Fe} / \mathrm{H}]$ abundances from the $\Delta \mathrm{S}$ method, independently of the pulsation phase.

\section{METALLICITY DISTRIBUTION}

We already mentioned that we are dealing with a sample of 2,903 RRLs on the same metallicity scale. Note that for objects that belong to different data sets we are adopting the following priority. The iron abundances based on the Magurno et al. (2018) sample (104) were included with their original estimates and intrinsic errors. For the RRLs for which the error was not provided, we assumed a mean error of 0.1 dex. The original iron abundances were also included for the five cluster RRLs and the six RRLs retrieved from the Rave DR5 catalogue. The former sample has an intrinsic error of 0.15 dex, while the latter one has an intrinsic error of 0.20 dex. The RRLs for which the iron abundance is based on the current $\Delta S$ method $(2,382)$ come immediately after in the priority list, and the error for this sample was assumed equal to the standard deviation of the calibration with the HR metallicity scale (0.29 dex). This is the largest and most homogenous sample of RRL iron abundances ever estimated. These two samples were complemented with RRL iron abundances provided by Sesar et al. (2013b, 50 stars), by Dambis et al. (2013, 360 stars) and by Duffau et al. (2014, 57 stars). For these samples the error on individual measurements was estimated by assuming a mean error of 0.15 , of 0.22 and 0.15 dex, respectively. Finally, we added the RRL iron abundances provided by the SDSS-SSPP survey (65). The error on individual measurements were estimated by summing in quadrature the original uncertainties FEHADOPUNC with the standard deviation of the calibration with the $\mathrm{HR}$ metallicity scale 
Table 1. Number of objects per dataset included in the iron catalogue.

\begin{tabular}{|c|c|c|c|c|c|c|c|c|c|}
\hline & SEGUE & Magurno+18 & NGC 5272 & Rave DR5 & Sesar +13 & Dambis +13 & Duffau +14 & SSPP & $\epsilon[\mathbf{F e} / \mathbf{H}]$ \\
\hline SEGUE & 2382 & 0 & 5 & 0 & 21 & 6 & 18 & 0 & 0.29 \\
\hline Magurno+18 & $\ldots \ldots \ldots$ & 104 & 0 & 0 & 0 & 72 & 0 & 0 & 0.10 \\
\hline NGC 5272 & $\ldots \ldots$ & $\ldots$ & 5 & 0 & 0 & 0 & 0 & 0 & 0.15 \\
\hline Rave DR5 & $\ldots \ldots \ldots$ & $\ldots \ldots \ldots \ldots$ & $\ldots \ldots \ldots \ldots$ & 6 & 0 & 2 & 0 & 0 & 0.20 \\
\hline Sesar+13 & $\ldots \ldots$ & $\ldots \ldots \ldots \ldots \ldots$ & $\ldots \ldots \ldots$ & $\ldots \ldots \ldots$ & 50 & 0 & 0 & 1 & 0.15 \\
\hline Dambis +13 & $\ldots \ldots \ldots$ & $\ldots \ldots \ldots \ldots$ & $\ldots \ldots \ldots$ & $\ldots \ldots \ldots \ldots$ & $\ldots \ldots \ldots$ & 360 & 0 & 0 & 0.22 \\
\hline Duffau+14 & $\ldots \ldots$ & $\ldots \ldots \ldots \ldots \ldots$ & $\ldots \ldots \ldots \ldots$ & $\ldots \ldots \ldots$ & $\ldots \ldots \ldots$ & $\ldots \ldots \ldots \ldots$ & 57 & 1 & 0.15 \\
\hline SSPP & $\ldots \ldots$ & $\ldots \ldots \ldots \ldots$ & $\ldots \ldots \ldots$ & $\ldots \ldots \ldots$ & $\ldots \ldots \ldots$ & $\ldots \ldots \ldots \ldots$ & $\ldots \ldots \ldots \ldots$ & 65 & FEHADOPUNC $a$ \\
\hline
\end{tabular}

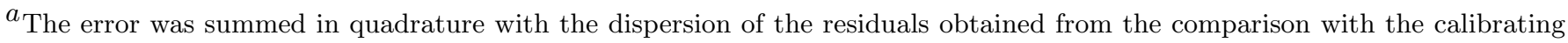
sample $(0.27$ dex $)$.

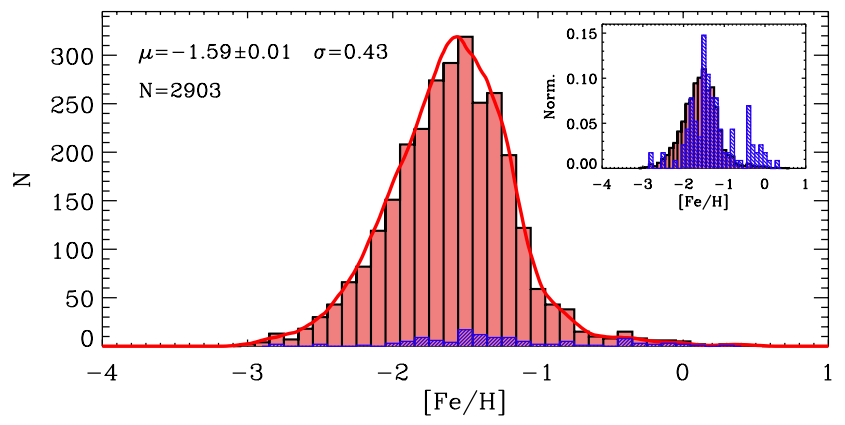

Figure 12. Metallicity distribution of the entire spectroscopic sample (in red) and for the high resolution sample (blue histogram). The orange line shows the smoothed metallicity distribution. The inset shows the same metallicity distributions, but area normalised.

(0.27 dex, see Sect. 4.1). The final iron abundance of RRLs in common among different medium/low resolution data sets $(\Delta \mathrm{S}$, Sesar et al. 2013b, Dambis et al. 2013, Duffau et al. 2014 and SSPP) was estimated as the mean of the different measurements and the errors were summed in quadrature. In Table 1 are listed the number of stars in common between the different datasets.

Fig. 12 shows the metallicity distribution of the entire RRab spectroscopic sample (red histogram) together with the smoothed metallicity distribution (orange line). The latter one was smoothed using a Gaussian kernel with unitary weight and $\sigma$ equal to the error of the individual estimates. The mean and the standard deviation of the smoothed distribution are also labelled. Data plotted in this figure bring forward several interesting features worth being discussed in detail.

a) Mean and Standard Deviation - The spectroscopic sample we are dealing with is more than a factor of five larger than any previous spectroscopic investigation of field RRLs (Dambis et al. 2013; Kinman et al. 2012; Layden 1993, 1994, 1995). The current mean metal abundance agrees quite well, within the errors, with similar estimates available in the literature $([\mathrm{Fe} / \mathrm{H}]=-1.59$ vs -
1.65, Layden 1994). The same outcome applies to the standard deviation, indeed, the difference $(\sigma=0.43$ vs 0.34 dex $)$ is once again marginal if we take account for the difference in the sample size.

b) Tails - The metallicity distribution appears more skewed toward the metal-poor regime, indeed, the metal-poor tail approaches $[\mathrm{Fe} / \mathrm{H}] \simeq-3$, while the metalrich one approaches Solar iron abundance. The quoted metallicity range is also supported by iron abundances based on high-resolution (HR) spectra (blue dashed areas). The main difference between the HR abundances and those based on lower-resolution (LR) spectra is that the former ones show a more prominent metal-rich tail and a less prominent metal-poor tail when compared with the latter ones. A glance at the metallicity distributions plotted in the inset of the same figure, that are normalised according to the area, shows even more clearly the difference in the metal-poor/metal-rich tails. On the basis of the current data it is not clear whether the metal-poor tail based on LR spectra might be a drift of the current absolute calibration or intrinsic. Note that the metallicity regime more metal-poor than $[\mathrm{Fe} / \mathrm{H}] \sim-$ 2.3 is not covered by cluster RRLs and the number of field, very metal-poor RRLs for which iron abundances is based on HR spectra is still limited (four). This limitation applies if we also take account for RRc variables.

c) Magnitude distribution - The HR sample is only limited to bright nearby RRLs, while the whole sample covers more than $120 \mathrm{kpc}$ (see Fig. 2). This indicates that the difference in the metallicity distribution between iron abundances based on either HR spectra or $\Delta \mathrm{S}$ method might also be caused by an observational bias affecting the former sample.

\section{THE FINE STRUCTURE OF THE BAILEY DIAGRAM}

The reasons why the Bailey diagram is a useful diagnostic to investigate the pulsation properties of variable stars have already been mentioned in the Sect. 1 . 
Here we only mention two relevant key points: $a$ ) it is independent of uncertainties affecting distance and reddening; b) cluster RRLs can be split into two groups called "Oosterhoff I" (OoI, mean RRab period of 0.55 days) and "Oosterhoff II" (OoII. mean RRab period of 0.65 days). The pioneering investigations concerning the metal content of globular clusters by Arp (1955) and by Kinman (1959) clearly demonstrated that OoI globulars are more metal-rich than OoII globulars. A quantitative investigation of the dependence of the Oosterhoff dichotomy on the metal content has been hampered by two intrinsic properties of Galactic globulars:

a) The metallicity distribution of Galactic globulars is bimodal (Harris 1991) with a well defined minimum for $[\mathrm{Fe} / \mathrm{H}]=-0.8 /-1.0$. Moreover, the metal-poor tail does not approach the limit of field Halo stars, while the metal-rich tail does not approach the limit of old metalrich Bulge stars. The difference is well known and it is tightly connected with the formation mechanism of globular clusters (Choksi et al. 2018).

b) Galactic globulars display at fixed metal content relevant changes in the horizontal-branch (HB) morphology, the so-called "second parameter" problem. This means that at fixed metal-content they might or they might not host RRLs according to their HB morphology. Nearby dwarf galaxies do not help in unraveling the skein, because their HB morphologies are quite similar. Indeed, only a few of them host an old stellar component that is either more metal-rich than $[\mathrm{Fe} / \mathrm{H}]=-1.0$ or more metal-poor than $[\mathrm{Fe} / \mathrm{H}]=-2.2$ (see Fig. 12 in McConnachie 2012). Quite often these stellar systems have been classified as Oosterhoff intermediate, i.e. the RRab attain mean periods that are between OoI and OoII clusters. The reader interested in detailed discussion concerning the difference among different globulars and nearby dwarf galaxies is referred to Fiorentino et al. (2017) and Braga et al. (2018), and references therein.

The quoted circumstantial evidence indicates that we still lack a homogenous and detailed analysis of the Bailey diagram as a function of the metal content. In this context it is worth mentioning that we are neglecting the metallicity estimates based either on photometric indices such as the inversion of the PL relation (Braga et al. 2016; Martínez-Vázquez et al. 2016; Bono et al. 2019 ) or the Fourier decomposition of the light curve (Jurcsik \& Kovacs 1996; Nemec et al. 2013; Elorrieta et al. 2016; Hajdu et al. 2018). Data plotted in Fig. 13 open a new path concerning the dependence of the luminosity amplitude on metallicity.

a) Period-metallicity correlation - The metallicity is colour coded (see the bar on the right axis) and indicates that RRab variables become, on average, steadily

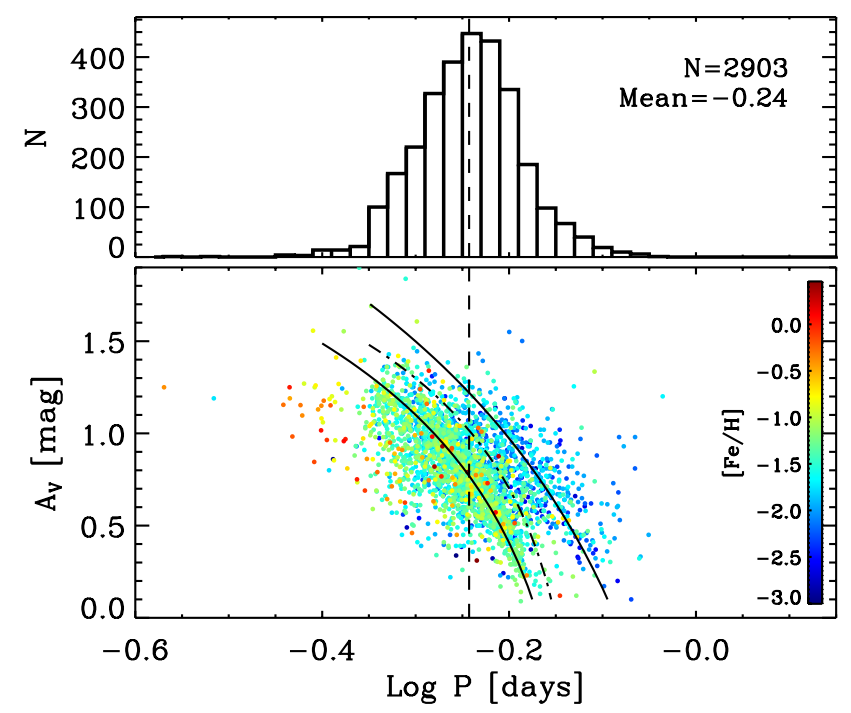

Figure 13. Top: Period distribution of the entire spectroscopic sample. Bottom: Bailey diagram of the spectroscopic sample. The metallicity is colour coded and the colour bar is plotted on the right. The vertical dashed line marks the mean period of the entire sample. The solid lines display the new analytical relations for OoI and OoII overdensities. The dot-dashed line shows the Oosterhoff intermediate loci, defined as the "valley" between the two main overdensities.

more metal-rich when moving, at fixed amplitude, from the long- to the short-period regime. The trend was already known, but the current data are suggesting that the variation of the mean period of RRab variables is continuous, i.e. the distribution of the RRab variables in the Bailey diagram is not uniform, but the variation is far from being dichotomic. To trace the key features of the Bailey diagram we produced a 3D histogram $\left(A_{V}\right.$, $\log \mathrm{P}$, number of RRLs) with the entire RRL sample. We traced the local maxima and the local minima in this $3 \mathrm{D}$ diagram and then we smoothed them by applying a running average. The two solid lines display the "mean" locus of the local maxima associated to OoI and OoII clusters, while the dashed line traces the Oosterhoff intermediate loci, defined as the local minima between the two main overdensities. The analytical relations for the three Oosterhoff sequences are the following:

$$
\begin{aligned}
\text { OoI }: A_{V} & =2.62+2.08 \cdot \log (-0.11-\log P) \\
\text { OoII }: A_{V} & =3.13+3.48 \cdot \log (0.041-\log P) \\
\text { OoInt }: A_{V} & =2.57+1.72 \cdot \log (-0.12-\log P)
\end{aligned}
$$

The quoted relations are in good agreement with similar relations for OoI and OoII groups provided by Zorotovic et al. (2010) and based on cluster RRLs collected by Cacciari et al. (2005). The mean difference in luminosity amplitude, over the entire period range, is 


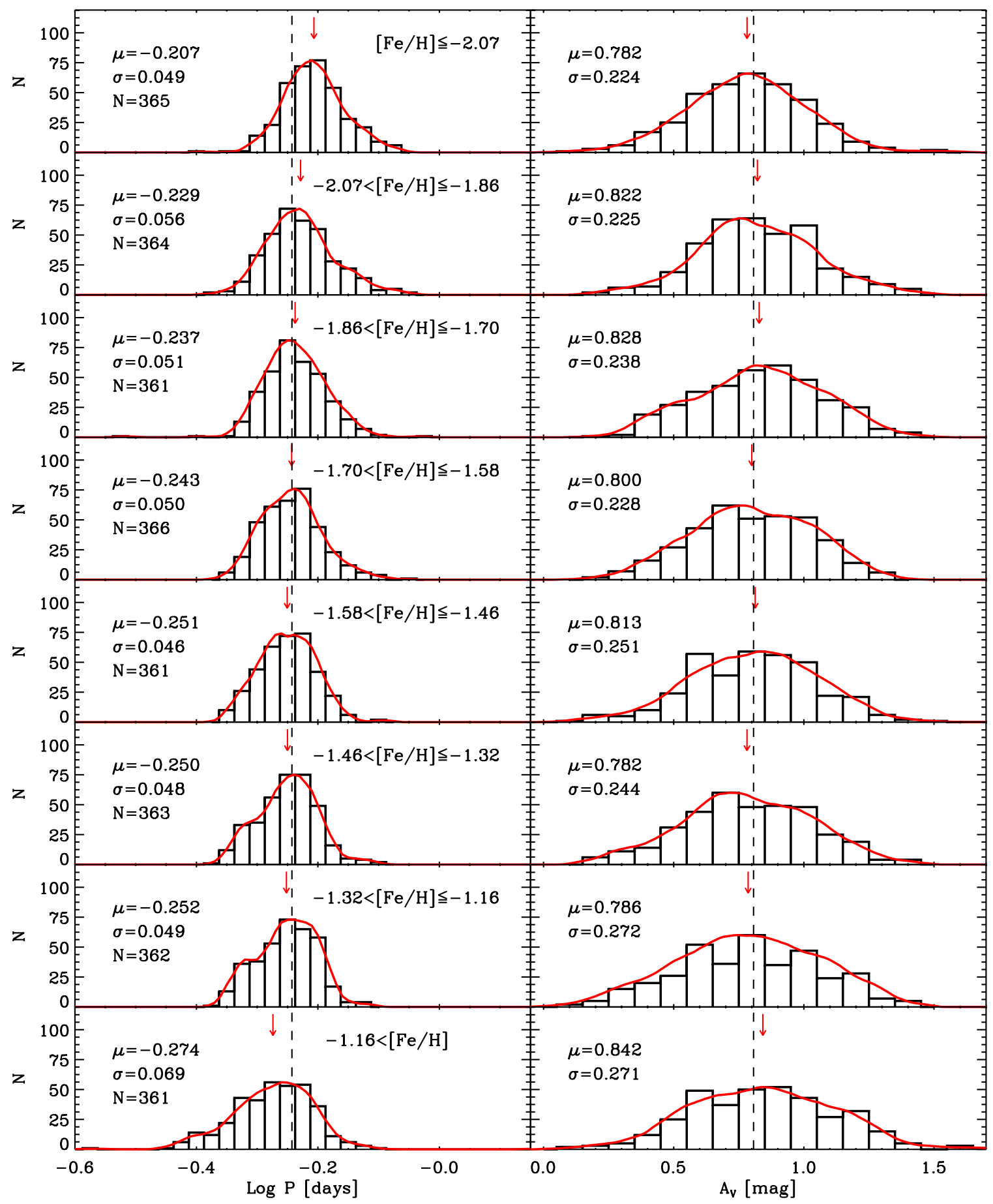

Figure 14. Period (left panels) and visual amplitude (right panels) distributions of the spectroscopic sample. The red lines display the smoothed distributions. The sample was split in eight metallicity bins including a similar number of objects (see labelled values). The red arrows mark the mean period and the mean amplitude of the individual bins. The dashed lines show the mean $\log P$ and mean $A_{V}$ of the total sample. 
$\sim 0.2$ mag for the OoI group and $\sim 0.1$ mag for OoII group.

Note that the Oo sequences are far from being parallel when moving from the short- to the long-period regime. Moreover, the region of the Bailey diagram among the two Oo sequences with periods of the order of $\log P \sim-0.15$ and amplitudes smaller than 0.5 mag appears empty. This suggests that this region is a sort of avoidance region for a broad range of metal abundances.

b) Period-amplitude-metallicity correlation - The RRab cover, at fixed metallicity and luminosity amplitude, a broad period range. This means that period, amplitude and metallicity do not obey to simple linear correlations.

To constrain on a more quantitative basis the variation of the pulsation properties (period, luminosity amplitude) as a function of the metal content we divided the entire spectroscopic sample in eight different metallicity bins. The edges of the individual metallicity bins (see labeled values in Fig. 14) were changed in such a way that they include a similar number of RRab variables. The left panels of Fig. 14 display from top to bottom the period distribution of RRab variables from the metal-poor to the metal-rich tail. The mean period, the standard deviation and the number of RRLs per metallicity bin are also labeled. The right panels show the $V$ amplitude distributions of the same RRLs plotted in the left panels. The period and the amplitude distributions display several interesting features:

a) The mean period becomes systematically shorter when moving from the metal-poor to the metal rich regime. Indeed, the red arrow moves from the right to the left of the mean period of the entire sample (vertical dashed line).

b) The period distribution is asymmetric over the entire metallicity range, but the skewness of the distribution moves from the long to the short period range when moving from the metal-poor to the metal-rich regime. The standard deviation of the different period distribution is quite constant, but the period distribution in the metal-rich regime becomes steadily flatter.

c) The luminosity amplitudes do not display the linear trend found for the pulsation periods. Indeed, the mean luminosity amplitude shows a modest variation and it moves either to slightly smaller or to slightly larger values of the mean global amplitude in the different metallicity bins. This evidence is suggesting that the dependence of the luminosity amplitude appears to be significantly milder than the dependence of the pulsation period. In passing, we also note that the large amplitude tail becomes, as expected, more and more relevant in the metal-rich regime (HASPs).

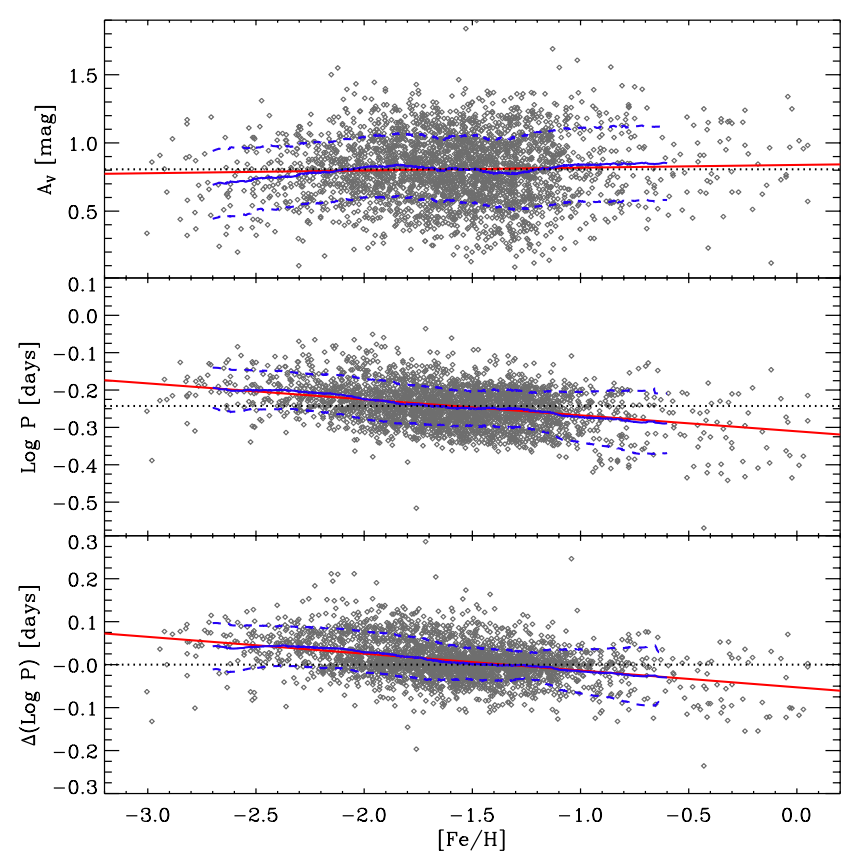

Figure 15. Top: $V$ amplitude as function of $[\mathrm{Fe} / \mathrm{H}]$. A running average (blue) and a linear regression (red) are also displayed. Middle: The same as the top but with the $\log P$ on y-axis. Bottom: The same as the top but with the $\Delta \log P$ on y-axis, i.e. the difference in period with the OoI relation. The horizontal dotted lines mark the mean values of $y$-axis

To overcome the limitation in the number of metallicity bins and possible subtle fluctuations in correlation between the two pulsation parameters and the metallicity we performed a running average. The entire sample of RRab variables was ranked as a function of the metal content and we estimated the running average, with a running box containing 500 objects. Note that in this estimate we neglected the very metal-poor $([\mathrm{Fe} / \mathrm{H}] \leq-2.7)$ and the very metal-rich $([\mathrm{Fe} / \mathrm{H}] \geq-0.4)$ tail, due to the poor statistics in these metallicity ranges. The metallicity and the mean visual amplitude of the bin were estimated as the mean over the individual iron abundances and visual amplitudes of the 500 objects included in the box. We estimated the same quantities moving by one object in the ranked list until we took account for the last object in the sample with the most metal-rich abundance. The solid blue line plotted in the top panel of Fig. 15 shows the running average, while the two dashed lines display the $1 \sigma$ standard deviation.

The linear fit (red line) plotted in the same panel shows a mild increase in the visual amplitude when moving from the metal-poor to the metal-rich regime. The linear relation fitting the data is the following:

$$
A_{V}=0.84( \pm 0.02)+0.02( \pm 0.01) \cdot[\mathrm{Fe} / \mathrm{H}]
$$


However, the difference with the mean global amplitude (horizontal dotted line) is of the order of a few hundredths of a magnitude. Indeed, the current fit suggests that a variation of $\approx 2$ dex in metallicity causes a variation of $\sim 0.04$ magnitudes in visual amplitude. The current findings clearly indicate that the association of a luminosity amplitude to an iron abundance should be cautiously treated, indeed, at fixed visual amplitude, field RRLs cover more than 2 dex in metal content.

The dependence of the mean period on the iron abundance is more solid, and indeed, the mean period decreases from 0.63 days in the metal-poor regime $([\mathrm{Fe} / \mathrm{H}] \sim-2.5)$ to 0.51 days in the metal-rich $([\mathrm{Fe} / \mathrm{H}] \sim-$ $0.5)$. This means a steady decrease of 0.12 days over a variation of 2 dex in metallicity. We evaluated the running average values (blue solid line in the middle panel of Fig. 15) and performed a linear fit (red line) finding:

$$
\log P=-0.311( \pm 0.004)-0.044( \pm 0.002) \cdot[\mathrm{Fe} / \mathrm{H}]
$$

The linear variation of the mean period as a function of the metallicity and the similarity of the standard deviation over the entire sample is further supporting the smooth variation of this intrinsic parameter when moving from the metal-poor to the metal-rich regime. To constrain on a more quantitive basis possible variations among metal-poor, metal-intermediate and metal-rich regime, we also estimated the difference in period between individual RRLs and the Oo I analytical relation derived in Sect. 6. Data plotted in the bottom panel of Fig. 15 show, once again, a smooth variation over the entire metallicity range, with the following linear relation:

$$
\Delta \log P=-0.054( \pm 0.003)-0.040( \pm 0.002) \cdot[\mathrm{Fe} / \mathrm{H}]
$$

The current iron abundances allow us to investigate the correlation existing between the Oosterhoff types and metal content. In Fig. 16 we selected on the Bailey diagram the candidate RRLs for OoI, OoII and OoInt around the Oosterhoff loci defined above. To overcome possible spurious effects concerning the size of the subsamples, the thickness of the regions around Oosterhoff loci were selected in order to provide a similar number of RRLs. The left panels of Fig. 16 show the selections we made for OoI (top in red), OoInt (middle in green) and OoII (bottom in blue) variables, while the right panels of the same figure display the related metallicity distributions over-imposed to the global RRLs distribution (grey solid area). The metallicity trend is clear, showing a more metal-rich distribution for the OoI, with a mean iron abundance of $[\mathrm{Fe} / \mathrm{H}]=-1.46$, to a more metal-poor distribution for the OoII, with a mean iron abundance of $[\mathrm{Fe} / \mathrm{H}]=-1.88$.
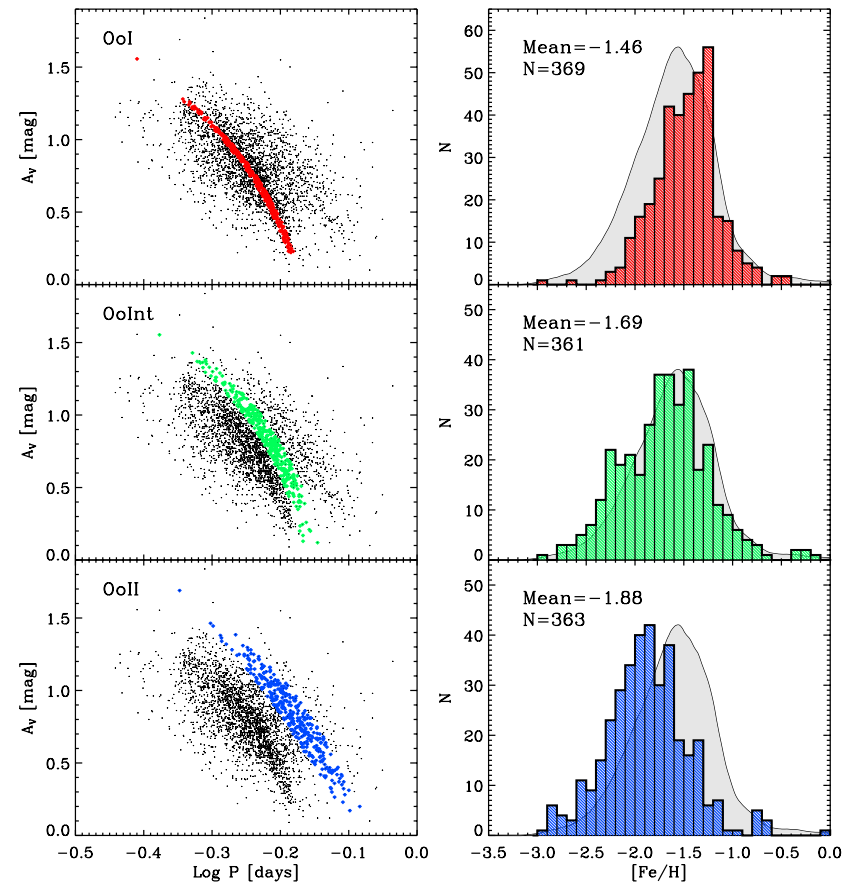

Figure 16. Bailey diagrams (left panels) and metallicity distributions (right panels) for OoI, OoInt and OoII samples. The grey solid area shows the distribution, normalised by the total area, of the the entire spectroscopic sample.

The current findings are supporting the empirical evidence concerning the variation of the mean period as a function of the metallicity brought forward long ago by Arp (1955) and by Kinman (1959). It is also supporting the period variation suggested by Sandage in a series of papers (Sandage 1981a,b, 1982). However, it is also suggesting that the Oosterhoff dichotomy is caused by the circumstantial evidence that metal-intermediate Galactic globulars either lack or only host a few RRLs (the prototype is M13, Castellani 1983; Renzini 1983). This means that it is not directly connected either with an evolutionary or with a pulsation property of RRLs. The gap in the mean period between OoI and OoII globulars appear to be the consequence of the GC diversity.

It is worth mentioning that the hysteresis mechanism was suggested more than 40 years ago by van Albada \& Baker (1973) to explain the difference between OoI and OoII clusters as a variation in the period distribution across the so-called OR" region, i.e. the region of the instability strip in which the variables can pulsate either in the fundamental or in the first overtone or in both of them (Bono \& Stellingwerf 1994). On the basis of the current findings we cannot exclude that the hysteresis mechanism might affect the period distribution across the instability strip, but its role in explaining the Oosterhoff dichotomy appears marginal. These working hypotheses are not new, they were originally suggested by 
Castellani (1983) and Renzini (1983) in two contributed papers.

To give them due credits we decided to quote the paragraphs in which they addressed this specific issue.

Concerning the Oosterhoff effect, it now appears that there is a real gap in $[\mathrm{Fe} / \mathrm{H}]$ between the two Oosterhoff types, and the famous discontinuity in $\left\langle P_{a b}\right\rangle$ naturally follows from the Sandage's relation: $\Delta \log P=$ $-0.06 \Delta[\mathrm{Fe} / \mathrm{H}]$. Indeed, BHB clusters with $\langle[\mathrm{Fe} / \mathrm{H}]\rangle=-$ 1.8 , just fill the gap between Oo. type I and II clusters found by Sandage (cf. Fig. 4 in Sandage 1982). In other words, the Oosterhoff effect is a consequence of the nonmonotonic behaviour of the $\mathrm{HB}$ with respect to $[\mathrm{Fe} / \mathrm{H}]$." (Renzini 1983).

This does not exclude that an hysteresis mechanism is acting. It only suggests that at the origin of the different behaviour of the two classes there is a discontinuity in the evolutionary parameters of the clusters. Either one accepts a real discontinuity in the history of Galactic $G C s$, or one concludes that clusters connecting OoI and OoII do exist, but they have no RR Lyrae." (Castellani 1983).

\section{STELLAR POPULATION COMPARISONS}

The new spectroscopic sample allows us to investigate on a more quantitative basis the difference in the Bailey diagram and in the period distribution between Halo RRLs and RRLs in nearby stellar systems. The candidate Halo stars $(2,354)$ were separated from the RRLs belonging to the Sagittarius stream by using the spatial over-density" criterium discussed in Sect. 2. Data plotted in the panel a) of Fig. 17 display the comparison of the period distribution between the current spectroscopic sample (grey shaded area) and the Bulge RRLs (OGLEIV, Soszyński et al. 2014, orange shaded area). The panel f) shows the same comparison, but in the Bailey diagram, and the Bulge RRLs are marked with orange dots, while the Halo spectroscopic sample with a black contour (95\% level). The empirical evidence indicates that the period distribution of Bulge RRLs is systematically shorter than Halo RRLs. Moreover, the short period tail is significantly more relevant in the Bulge sample than in the Halo. This evidence together with a sizeable sample of HASPs RRLs (Fiorentino et al. 2015) is suggesting that the metallicity distribution of Bulge RRLs is systematically more metal-rich than Halo RRLs.

Panels b) and g) show the comparison between the spectroscopic sample and cluster RRLs. The difference concerning the occurrence of the Oosterhoff gap has already been discussed in Sect. 6. Here we only mention the large number of metal-rich RRLs present in the field when compared with Galactic globulars. The presence of metal-rich RRLs has been considered for several decades a conundrum" (Kraft 1972; Taam et al. 1976; Smith 1984), because metal-rich $([\mathrm{Fe} / \mathrm{H}] \geq-0.7)$ globular clusters do not host RRLs. This problem was partially alleviated, by the discovery of sizeable sample of RRLs in the two metal-rich globulars NGC 6441 and NGC 6338 (Pritzl et al. 2000). The occurrence of RRLs at Solar metal abundance dates back to Smith (1984) and to Walker \& Terndrup (1991), however, these investigations were based on low-resolution spectroscopy ( $\Delta \mathrm{S}$ method). Only recently, Sneden and collaborators measured roughly 30 RRLs at Solar metal content by using high resolution spectra (Chadid et al. 2017; Sneden et al. 2018). The current findings are soundly supporting this result and indicate that the lack of RRLs in Bulge, metal-rich globulars is mainly an observational bias. The next Gaia data release, including accurate estimates of both proper motion and geometrical distances, will allow us to shed new lights on the possible occurrence of metal-rich cluster RRLs.

The agreement between the current sample and Large Magellanic Cloud (LMC) RRLs (panels c) and h)) is quite interesting and fully supports the results obtained by Fiorentino et al. $(2015,2017)$ based on the entire sample of Halo RRLs known at that time $(\sim 45,000)$. They found a strong similarity both in the period distribution and in the Bailey diagram between Halo and LMC RRLs and suggested that this is a sound independent support for the major merging scenario (Tissera et al. 2014; Zolotov et al. 2009). It is worth mentioning that the metallicity distribution of LMC RRLs has been investigated by Clementini et al. (2003). They found an average metal abundance of $[\mathrm{Fe} / \mathrm{H}] \sim-1.48$ and the metallicity distribution ranges from -2.1 to -0.5 dex. The current similarity between Halo and LMC RRLs is suggesting that the latter sample might cover a broader metallicity range.

The comparison between the spectroscopic sample and the Small Magellanic Cloud (SMC) RRLs (panels d) and i)) shows quite clearly that the former sample includes a tail of metal-rich RRLs that is not present at all in the SMC. Indeed, the lack of HASPs is evident both in the period distribution and in the Bailey diagram. There is only one SMC globular with an age larger than ten Gyrs hosting RRLs, that is NGC 121 (Walker \& Mack 1988; Fiorentino et al. 2008) and it is once again metal intermediate $([\mathrm{Fe} / \mathrm{H}] \sim-1.28$, Dalessandro et al. 2016). The difference between SMC and LMC is expected, since the former stellar system is significantly less massive than the latter one. This means that the chemical enrichment has been less efficient in the SMC than in the LMC. This 

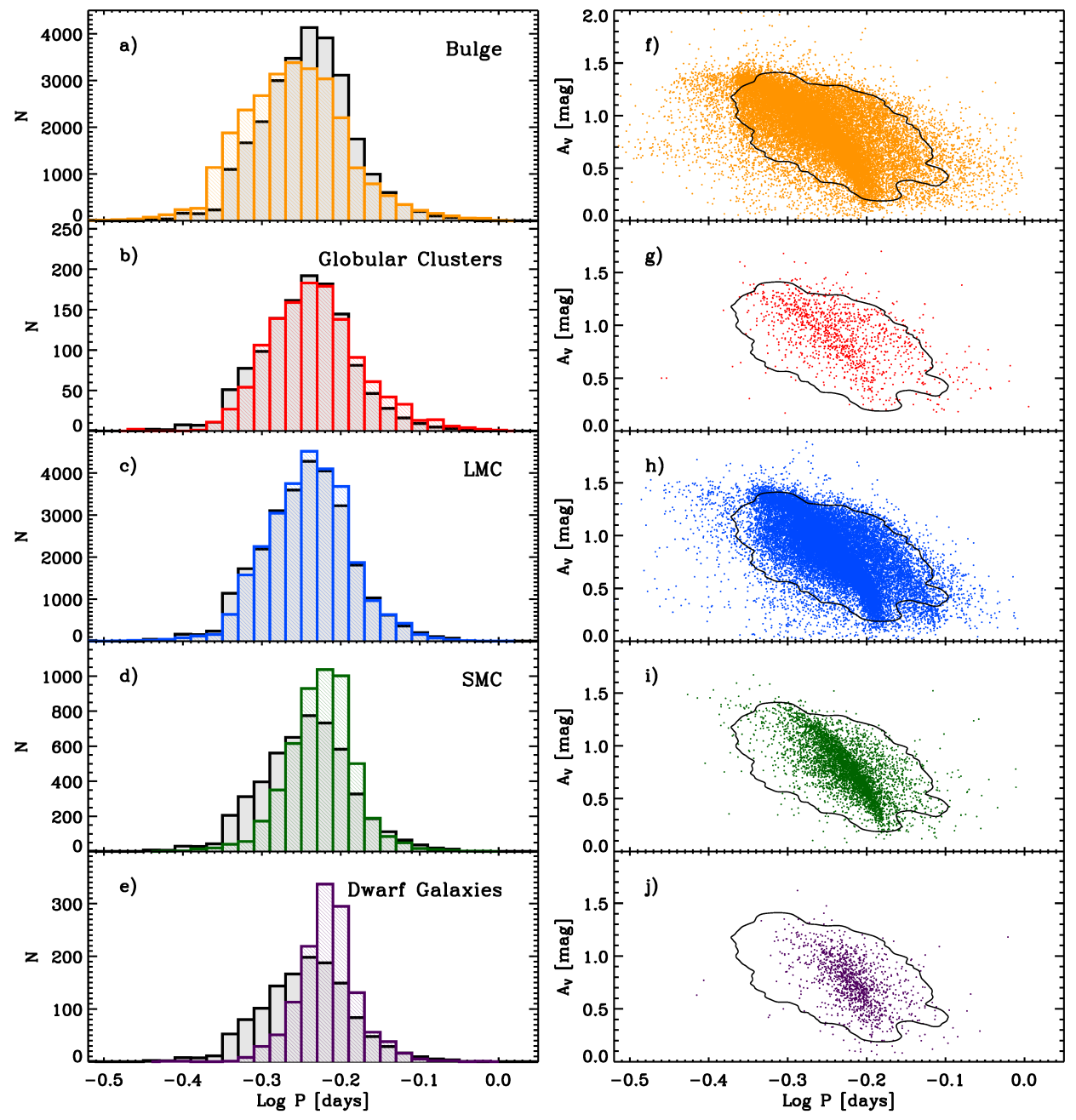

Figure 17. Period distribution (left panels) and Bailey diagrams (right panels) of the Halo RRab sample compared with different stellar systems. The grey contours and histograms show the location and distribution (normalised by the total area) of the Halo spectroscopic sample $(2,354)$.

is a consequence of the Mass-Metallicity scaling relation (Chilingarian et al. 2011).

The role played by the total baryonic mass in the chemical evolution becomes even more relevant in the comparison with RRLs in nearby gas poor dwarf galaxies (panels e) and j) purple shaded area). The RRLs in gas-poor dwarf galaxies adopted in the current investigation come from the same sample selected by Braga et al. (2016). These stellar systems only include a handful of HASPS, i.e. RRab with periods shorter than $\approx 0.5$ days. The lack of a sizeable sample of longperiod, metal-poor RRLs is also quite clear. This is the double circumstantial evidence causing gas poor dwarf galaxies to be Oosterhoff intermediate". We have already discussed in Sect. 1 the metallicity distribution of nearby dwarf galaxies, but we would like to add a few words of caution in using it. Current spectroscopic measurements mainly rely on high/medium resolution spec- troscopy of red giants (APOGEE, Majewski et al. 2017, GALAH Buder et al. 2018). The spectroscopic measurements for the stellar systems with multiple star formation episodes are an average of old- and intermediateage stellar populations. This is a consequence of the so-called age-metallicity degeneracy along the red-giant branch. The consequence is that red-giant stars with old/intermediate-age progenitors and different metallicities attain similar magnitudes and colours along the red giant branch. A novel approach to overcome this problem was recently suggested by Monelli et al. (2014) based on a photometric index $c_{\mathrm{U}, \mathrm{B}, \mathrm{I}}=[(U-B)-(B-I)]$, but it has only been applied to the Carina dwarf spheroidal galaxy (Fabrizio et al. 2016) and indicates that the peak of the old stellar population associated with RRLs is systematically more metal-poor than the intermediate-age one associated with red clump stars. New and accurate spectroscopic measurements are required to fully investi- 
gate the RRLs in gas-poor dwarf galaxies. In passing, we also note that nearby dwarf galaxies always host RRLs and the morphology of the $\mathrm{HB}$ is dominated neither by hot/extreme HB stars nor by red HB stars (Bono et al. 2016). Moreover, there is no evidence of the occurrence of a second parameter" problem among gas-poor dwarf galaxies.

\section{CONCLUSIONS}

The last twenty years have been quite crucial for the understanding of stellar populations and evolutionary properties of low-, intermediate- and high- mass stars. This relevant step forward applies not only to Galactic stellar populations, but also to resolved stellar populations in Local Group and Local Volume galaxies. In spite of these indisputable advantages, several longstanding astrophysical problems still await for a quantitative explanation of the different physical mechanisms and input parameters driving their occurrence. The Oosterhoff dichotomy is among them. Several working hypotheses have been suggested in the literature, but the lack of accurate and homogeneous metal abundances hampered a solid explanation for the occurrence of this phenomenon. In particular, we were lacking firm clues concerning the role that the environment plays in explaining the basics of the Bailey diagram. In this investigation we estimated new and homogeneous iron abundances for a sample of 2,382 field RR Lyrae stars by using medium resolution SDSS-SEGUE spectra. They were complemented with estimates available in the literature and based either on high, or on intermediate or on low spectral resolutions. We ended up with a sample of 2,903 RRLs, the largest and most homogenous sample of iron abundances ever estimated for fundamental RRLs. The results we found are summarised in the following.

- The $\Delta \mathrm{S}$ approach adopted to derive the iron abundances was also validated for a fundamental field RRL (V Ind) for which we collected X-shooter spectra covering the entire pulsation cycle. The iron estimates agree, within the errors, on the whole pulsation period, including also the critical part of the rising branch.

- We found a metallicity distribution slightly skewed toward the metal-poor regime, with a mean iron abundance of $[\mathrm{Fe} / \mathrm{H}]=-1.59 \pm 0.01$ and a dispersion of 0.43 dex.

- The RRL plotted in the Period-Amplitude plane (Bailey diagram) allow us to define the period-amplitude relations for the three Oosterhoff sequences (OoI, OoII and OoInt) and to confirm the differences in metal content among these groups. Indeed, the OoI show an iron distribution more metal-rich $([\mathrm{Fe} / \mathrm{H}]=-1.46)$ than the OoInt $(-1.69)$ and the OoII $(-1.88)$.

- We were able to find a continuous and linear correlation between the metallicity and the period, confirming the theoretical and empirical evidence brought forward in the literature, indicating that the long-standing problem of the Oosterhoff dichotomy among Galactic globulars is the consequence of the lack of metal-intermediate clusters hosting RRLs.

- We compared the Halo RRLs period distribution and Bailey diagram with those of the nearby stellar systems. In particular, the Galactic Bulge and dwarf galaxies differ from the Halo, suggesting a metallicity distribution more metal-rich for Bulge stars against a more metalpoor distribution for dwarf galaxies.

In this context it is worth mentioning that the analytical relations we are providing for OoI, OoII and OoInt groups shall be applied to the mean period of sizeable RRL samples. The standard deviation in metal content, at fixed pulsation period, is too large to be applied to individual RRLs. The above findings indicate that the new spectroscopic sample is crucial to address a long-standing astrophysical problem. However, they should be cautiously treated, indeed, the current analysis is only based on fundamental RRab variables. A more comprehensive empirical scenario awaits for spectroscopic abundances of first overtone RRc variables.

\section{ACKNOWLEDGMENTS}

It is a real pleasure to thank the anonymous referee for her/his pertinent suggestions that improved the content and the readability of the paper. This work has made use of data from the European Space Agency (ESA) mission Gaia (https://www.cosmos.esa.int/gaia), processed by the Gaia Data Processing and Analysis Consortium (DPAC, https://www.cosmos.esa.int/web/ gaia/dpac/consortium). Funding for the DPAC has been provided by national institutions, in particular the institutions participating in the Gaia Multilateral Agreement. This research has made use of the GaiaPortal catalogues access tool, ASI - Space Science Data Center, Rome, Italy (http://gaiaportal.ssdc.asi.it). We would also like to acknowledge the financial support of INAF (Istituto Nazionale di Astrofisica), Osservatorio Astronomico di Roma, ASI (Agenzia Spaziale Italiana) under contract to INAF: ASI 2014-049-R.0 dedicated to SSDC. M.M. was partially supported by the National Science Foundation under Grant No. AST1714534. 


\section{REFERENCES}

Ahn, C. P., Alexandroff, R., Allende Prieto, C., et al. 2012, ApJS, 203, 21, doi: 10.1088/0067-0049/203/2/21

Akerlof, C., Amrose, S., Balsano, R., et al. 2000, AJ, 119, 1901, doi: 10.1086/301321

Alam, S., Albareti, F. D., Allende Prieto, C., et al. 2015, ApJS, 219, 12, doi: 10.1088/0067-0049/219/1/12

Allende Prieto, C., Sivarani, T., Beers, T. C., et al. 2008, AJ, 136, 2070, doi: 10.1088/0004-6256/136/5/2070

Arp, H. C. 1955, AJ, 60, 317, doi: 10.1086/107232

Asplund, M., Grevesse, N., Sauval, A. J., \& Scott, P. 2009, Annual Review of Astronomy and Astrophysics, 47, 481, doi: 10.1146/annurev.astro.46.060407.145222

Bailer-Jones, C. A. L., Rybizki, J., Fouesneau, M., Mantelet, G., \& Andrae, R. 2018, AJ, 156, 58, doi: 10.3847/1538-3881/aacb21

Bailey, S. I. 1902, Annals of Harvard College Observatory, 38

Beers, T. C. 1990, AJ, 99, 323, doi: 10.1086/115330

Bianchi, L., Shiao, B., \& Thilker, D. 2017, ApJS, 230, 24, doi: 10.3847/1538-4365/aa7053

Bickerton, S., Badenes, C., Hettinger, T., Beers, T., \& Huang, S. 2012, in IAU Symposium, Vol. 285, New Horizons in Time Domain Astronomy, ed. E. Griffin, R. Hanisch, \& R. Seaman, 289-290

Bono, G., Caputo, F., Cassisi, S., Castellani, V., \& Marconi, M. 1997, ApJ, 489, 822, doi: 10.1086/304807

Bono, G., Caputo, F., \& Marconi, M. 1995, AJ, 110, 2365, doi: 10.1086/117694

Bono, G., Caputo, F., \& Stellingwerf, R. F. 1994, ApJ, 423, 294, doi: 10.1086/173806

Bono, G., \& Stellingwerf, R. F. 1994, ApJS, 93, 233, doi: 10.1086/192054

Bono, G., Pietrinferni, A., Marconi, M., et al. 2016, Commmunications of the Konkoly Observatory Hungary, 105,149

Bono, G., Iannicola, G., Braga, V. F., et al. 2019, ApJ, 870, 115, doi: 10.3847/1538-4357/aaf23f

Braga, V. F., Stetson, P. B., Bono, G., et al. 2016, AJ, 152, 170, doi: 10.3847/0004-6256/152/6/170

—. 2018, AJ, 155, 137, doi: 10.3847/1538-3881/aaadab

Buder, S., Asplund, M., Duong, L., et al. 2018, MNRAS, 478, 4513, doi: 10.1093/mnras/sty 1281

Cacciari, C., Corwin, T. M., \& Carney, B. W. 2005, AJ, 129, 267, doi: 10.1086/426325

Caputo, F., Santolamazza, P., \& Marconi, M. 1998, MNRAS, 293, 364, doi: 10.1046/j.1365-8711.1998.01171.x

Cardelli, J. A., Clayton, G. C., \& Mathis, J. S. 1989, ApJ, 345, 245, doi: 10.1086/167900
Casagrande, L., \& VandenBerg, D. A. 2018, MNRAS, 479, L102, doi: 10.1093/mnrasl/sly104

Casey, A. R., Hawkins, K., Hogg, D. W., et al. 2017, ApJ, 840, 59, doi: 10.3847/1538-4357/aa69c2

Cassisi, S., Castellani, M., Caputo, F., \& Castellani, V. 2004, A\&A, 426, 641, doi: 10.1051/0004-6361:20041048

Castellani, M., Caputo, F., \& Castellani, V. 2003, A\&A, 410, 871, doi: 10.1051/0004-6361:20031381

Castellani, V. 1983, Mem. Soc. Astron. Italiana, 54, 141

Catelan, M. 2009, Ap\&SS, 320, 261, doi: 10.1007/s10509-009-9987-8

Chadid, M., Sneden, C., \& Preston, G. W. 2017, ApJ, 835, 187, doi: 10.3847/1538-4357/835/2/187

Chilingarian, I. V., Mieske, S., Hilker, M., \& Infante, L. 2011, MNRAS, 412, 1627, doi: 10.1111/j.1365-2966.2010.18000.x

Choksi, N., Gnedin, O. Y., \& Li, H. 2018, MNRAS, 480, 2343, doi: 10.1093/mnras/sty1952

Clement, C. M., Muzzin, A., Dufton, Q., et al. 2001, AJ, 122, 2587, doi: 10.1086/323719

Clementini, G., Cacciari, C., \& Lindgren, H. 1990, A\&AS, 85,865

Clementini, G., Gratton, R., Bragaglia, A., et al. 2003, AJ, 125, 1309, doi: 10.1086/367773

Clementini, G., Ripepi, V., Molinaro, R., et al. 2019, A\&A, 622, A60, doi: 10.1051/0004-6361/201833374

Coppola, G., Stetson, P. B., Marconi, M., et al. 2013, ApJ, 775, 6, doi: 10.1088/0004-637X/775/1/6

Coppola, G., Marconi, M., Stetson, P. B., et al. 2015, ApJ, 814, 71, doi: 10.1088/0004-637X/814/1/71

Cutri, R. M., \& et al. 2013, VizieR Online Data Catalog, 2328

Dalessandro, E., Lapenna, E., Mucciarelli, A., et al. 2016, ApJ, 829, 77, doi: 10.3847/0004-637X/829/2/77

Dall'Ora, M., Kinemuchi, K., Ripepi, V., et al. 2012, ApJ, 752, 42, doi: 10.1088/0004-637X/752/1/42

Dambis, A. K., Berdnikov, L. N., Kniazev, A. Y., et al. 2013, MNRAS, 435, 3206, doi: 10.1093/mnras/stt1514

Dekker, H., D'Odorico, S., Kaufer, A., Delabre, B., \& Kotzlowski, H. 2000, in Proc. SPIE, Vol. 4008, Optical and IR Telescope Instrumentation and Detectors, ed. M. Iye \& A. F. Moorwood, 534-545

Drake, A. J., Catelan, M., Djorgovski, S. G., et al. 2013a, ApJ, 763, 32, doi: 10.1088/0004-637X/763/1/32

-. 2013b, ApJ, 765, 154, doi: 10.1088/0004-637X/765/2/154

Drake, A. J., Graham, M. J., Djorgovski, S. G., et al. 2014, ApJS, 213, 9, doi: 10.1088/0067-0049/213/1/9 
Duffau, S., Vivas, A. K., Zinn, R., Méndez, R. A., \& Ruiz, M. T. 2014, A\&A, 566, A118, doi: 10.1051/0004-6361/201219654

Elorrieta, F., Eyheramendy, S., Jordán, A., et al. 2016, A\&A, 595, A82, doi: 10.1051/0004-6361/201628700

Evans, D. W., Riello, M., De Angeli, F., et al. 2018, A\&A, 616, A4, doi: 10.1051/0004-6361/201832756

Fabrizio, M., Nonino, M., Bono, G., et al. 2015, A\&A, 580, A18, doi: 10.1051/0004-6361/201525753

Fabrizio, M., Bono, G., Nonino, M., et al. 2016, ApJ, 830, 126, doi: 10.3847/0004-637X/830/2/126

Fernley, J., Barnes, T. G., Skillen, I., et al. 1998, A\&A, 330,515

Fiorentino, G., Contreras, R., Clementini, G., et al. 2008, arXiv e-prints. https://arxiv.org/abs/0801.2713

Fiorentino, G., Bono, G., Monelli, M., et al. 2015, ApJL, 798, L12, doi: 10.1088/2041-8205/798/1/L12

Fiorentino, G., Monelli, M., Stetson, P. B., et al. 2017, A\&A, 599, A125, doi: 10.1051/0004-6361/201629501

Freeman, K. C., \& Rodgers, A. W. 1975, ApJL, 201, L71, doi: 10.1086/181945

Gaia Collaboration, Brown, A. G. A., Vallenari, A., et al. 2018, A\&A, 616, A1, doi: 10.1051/0004-6361/201833051

Gratton, R., Sneden, C., \& Carretta, E. 2004, ARA\&A, 42, 385, doi: 10.1146/annurev.astro.42.053102.133945

Hajdu, G., Dékány, I., Catelan, M., Grebel, E. K., \& Jurcsik, J. 2018, ApJ, 857, 55, doi: 10.3847/1538-4357/aab4fd

Harris, W. E. 1991, ARA\&A, 29, 543, doi: 10.1146/annurev.aa.29.090191.002551

—. 2010, arXiv e-prints. https://arxiv.org/abs/1012.3224

Hoffman, D. I., Harrison, T. E., \& McNamara, B. J. 2009, AJ, 138, 466, doi: 10.1088/0004-6256/138/2/466

Jang, S., \& Lee, Y.-W. 2015, ApJS, 218, 31, doi: 10.1088/0067-0049/218/2/31

Jayasinghe, T., Kochanek, C. S., Stanek, K. Z., et al. 2018, MNRAS, 477, 3145, doi: 10.1093/mnras/sty838

Jurcsik, J., \& Kovacs, G. 1996, A\&A, 312, 111

Kinemuchi, K., Harris, H. C., Smith, H. A., et al. 2008, AJ, 136, 1921, doi: 10.1088/0004-6256/136/5/1921

Kinman, T. D. 1959, MNRAS, 119, 134, doi: 10.1093/mnras/119.2.134

Kinman, T. D., Cacciari, C., Bragaglia, A., Buzzoni, A., \& Spagna, A. 2007, MNRAS, 375, 1381, doi: 10.1111/j.1365-2966.2006.11394.x

Kinman, T. D., Cacciari, C., Bragaglia, A., Smart, R., \& Spagna, A. 2012, MNRAS, 422, 2116, doi: 10.1111/j.1365-2966.2012.20747.x

Kraft, R. P. 1972, in The Evolution of Population II Stars, ed. A. G. D. Philip, Vol. 4, 69-96
Kunder, A., Kordopatis, G., Steinmetz, M., et al. 2017, AJ, 153, 75, doi: 10.3847/1538-3881/153/2/75

Layden, A. C. 1993, PASP, 105, 1367, doi: 10.1086/133319

—. 1994, AJ, 108, 1016, doi: 10.1086/117132

—. 1995, AJ, 110, 2288, doi: 10.1086/117690

Lee, J.-W., \& Carney, B. W. 1999, AJ, 118, 1373, doi: $10.1086 / 301008$

Lee, Y. S., Beers, T. C., Sivarani, T., et al. 2008a, AJ, 136, 2022, doi: 10.1088/0004-6256/136/5/2022

—. 2008b, AJ, 136, 2050, doi: 10.1088/0004-6256/136/5/2050

Lee, Y.-W., Demarque, P., \& Zinn, R. 1990, ApJ, 350, 155, doi: 10.1086/168370

—. 1994, ApJ, 423, 248, doi: 10.1086/173803

Magurno, D. 2018, PhD thesis, University of Rome "Tor Vergata"

Magurno, D., Sneden, C., Braga, V. F., et al. 2018, ApJ, 864, 57, doi: 10.3847/1538-4357/aad4a3

Majewski, S. R., Skrutskie, M. F., Weinberg, M. D., \& Ostheimer, J. C. 2003, ApJ, 599, 1082, doi: $10.1086 / 379504$

Majewski, S. R., Schiavon, R. P., Frinchaboy, P. M., et al. 2017, AJ, 154, 94, doi: 10.3847/1538-3881/aa784d

Marconi, M., Bono, G., Pietrinferni, A., et al. 2018, ApJL, 864, L13, doi: 10.3847/2041-8213/aada17

Marconi, M., Nordgren, T., Bono, G., Schnider, G., \& Caputo, F. 2005, ApJL, 623, L133, doi: 10.1086/430273

Marconi, M., Coppola, G., Bono, G., et al. 2015, ApJ, 808, 50, doi: 10.1088/0004-637X/808/1/50

Marrese, P. M., Marinoni, S., Fabrizio, M., \& Altavilla, G. 2019, A\&A, 621, A144, doi: 10.1051/0004-6361/201834142

Marrese, P. M., Marinoni, S., Fabrizio, M., \& Giuffrida, G. 2017, A\&A, 607, A105, doi: 10.1051/0004-6361/201730965

Martínez-Vázquez, C. E., Monelli, M., Bono, G., et al. 2015, MNRAS, 454, 1509, doi: 10.1093/mnras/stv2014

Martínez-Vázquez, C. E., Stetson, P. B., Monelli, M., et al. 2016, MNRAS, 462, 4349, doi: 10.1093/mnras/stw1895

McConnachie, A. W. 2012, AJ, 144, 4, doi: 10.1088/0004-6256/144/1/4

McMahon, R. G., Banerji, M., Gonzalez, E., et al. 2013, The Messenger, 154, 35

Miceli, A., Rest, A., Stubbs, C. W., et al. 2008, ApJ, 678, 865, doi: 10.1086/533484

Monelli, M., Fiorentino, G., Bernard, E. J., et al. 2017, ApJ, 842, 60, doi: 10.3847/1538-4357/aa738d

Monelli, M., Milone, A. P., Fabrizio, M., et al. 2014, ApJ, 796, 90, doi: 10.1088/0004-637X/796/2/90 
Monson, A. J., Beaton, R. L., Scowcroft, V., et al. 2017, AJ, 153, 96, doi: 10.3847/1538-3881/153/3/96

Nemec, J. M., Cohen, J. G., Ripepi, V., et al. 2013, ApJ, 773, 181, doi: 10.1088/0004-637X/773/2/181

Nemec, J. M., Wehlau, A., \& Mendes de Oliveira, C. 1988, AJ, 96, 528, doi: 10.1086/114830

Oke, J. B., \& Gunn, J. E. 1982, PASP, 94, 586, doi: $10.1086 / 131027$

Oosterhoff, P. T. 1939, The Observatory, 62, 104

Pancino, E., Britavskiy, N., Romano, D., et al. 2015, MNRAS, 447, 2404, doi: 10.1093/mnras/stu2616

Petroni, S., Bono, G., Castellani, V., \& Marconi, M. 2004, in Frontiers of the Universe, ed. L. Celnikier \& J. T. Thanh van, 199

Pietrukowicz, P., Kozłowski, S., Skowron, J., et al. 2015, ApJ, 811, 113, doi: 10.1088/0004-637X/811/2/113

Pietrzyński, G., Graczyk, D., Gieren, W., et al. 2013, Nature, 495, 76, doi: 10.1038/nature11878

Pojmanski, G. 2002, AcA, 52, 397

Prada Moroni, P. G., Gennaro, M., Bono, G., et al. 2012, ApJ, 749, 108, doi: 10.1088/0004-637X/749/2/108

Preston, G. W. 1959, ApJ, 130, 507, doi: 10.1086/146743

Preston, G. W., \& Paczynski, B. 1964, ApJ, 140, 181, doi: 10.1086/147907

Pritzl, B., Smith, H. A., Catelan, M., \& Sweigart, A. V. 2000, ApJL, 530, L41, doi: 10.1086/312482

Pritzl, B. J., Smith, H. A., Stetson, P. B., et al. 2003, AJ, 126, 1381, doi: 10.1086/377024

Renzini, A. 1983, Mem. Soc. Astron. Italiana, 54, 335

Salaris, M. 2018, in The RR Lyrae 2017 Conference. Revival of the Classical Pulsators: from Galactic Structure to Stellar Interior Diagnostics, ed. R. Smolec, K. Kinemuchi, \& R. I. Anderson, Vol. 6, 21-28

Sandage, A. 1981a, ApJL, 244, L23, doi: 10.1086/183471

—. 1981b, ApJ, 248, 161, doi: 10.1086/159140

—. 1982, ApJ, 252, 553, doi: 10.1086/159582

—. 1990, ApJ, 350, 603, doi: 10.1086/168415

—. 1993, AJ, 106, 703, doi: 10.1086/116676

—. 2006, AJ, 131, 1750, doi: 10.1086/500012

—. 2010, ApJ, 722, 79, doi: 10.1088/0004-637X/722/1/79

Schlafly, E. F., \& Finkbeiner, D. P. 2011, ApJ, 737, 103, doi: 10.1088/0004-637X/737/2/103

Schlegel, D. J., Finkbeiner, D. P., \& Davis, M. 1998, ApJ, 500, 525, doi: $10.1086 / 305772$

Sesar, B., Ivezić, Ž., Stuart, J. S., et al. 2013a, AJ, 146, 21, doi: 10.1088/0004-6256/146/2/21

Sesar, B., Grillmair, C. J., Cohen, J. G., et al. 2013b, ApJ, 776, 26, doi: 10.1088/0004-637X/776/1/26
Shappee, B. J., Prieto, J. L., Grupe, D., et al. 2014, ApJ, 788, 48, doi: 10.1088/0004-637X/788/1/48

Skrutskie, M. F., Cutri, R. M., Stiening, R., et al. 2006, AJ, 131, 1163, doi: 10.1086/498708

Smee, S. A., Gunn, J. E., Uomoto, A., et al. 2013, AJ, 146, 32, doi: 10.1088/0004-6256/146/2/32

Smith, H. A. 1984, PASP, 96, 505, doi: 10.1086/131370

Sneden, C., Preston, G. W., Kollmeier, J. A., et al. 2018, AJ, 155, 45, doi: 10.3847/1538-3881/aa9f16

Soszyński, I., Udalski, A., Szymański, M. K., et al. 2014, AcA, 64, 177. https://arxiv.org/abs/1410.1542

Stetson, P. B., Fiorentino, G., Bono, G., et al. 2014, PASP, 126, 616, doi: 10.1086/677352

Stobie, R. S. 1971, ApJ, 168, 381, doi: 10.1086/151094

Storm, J., Carney, B. W., \& Latham, D. W. 1994, A\&A, 290, 443

Taam, R. E., Kraft, R. P., \& Suntzeff, N. 1976, ApJ, 207, 201, doi: 10.1086/154485

Tissera, P. B., Beers, T. C., Carollo, D., \& Scannapieco, C. 2014, MNRAS, 439, 3128, doi: 10.1093/mnras/stu181

Torrealba, G., Catelan, M., Drake, A. J., et al. 2015, MNRAS, 446, 2251, doi: 10.1093/mnras/stu2274

Valenti, E., Zoccali, M., Mucciarelli, A., et al. 2018, A\&A, 616, A83, doi: 10.1051/0004-6361/201832905

van Albada, T. S., \& Baker, N. 1973, ApJ, 185, 477, doi: $10.1086 / 152434$

van den Bergh, S. 1993a, MNRAS, 262, 588, doi: $10.1093 / \mathrm{mnras} / 262.3 .588$

—. 1993b, AJ, 105, 971, doi: 10.1086/116485

Vernet, J., Dekker, H., D’Odorico, S., et al. 2011, A\&A, 536, A105, doi: 10.1051/0004-6361/201117752

Vivas, A. K., Zinn, R., Abad, C., et al. 2004, AJ, 127, 1158, doi: $10.1086 / 380929$

Walker, A. R., \& Mack, P. 1988, AJ, 96, 872, doi: $10.1086 / 114853$

Walker, A. R., \& Terndrup, D. M. 1991, ApJ, 378, 119, doi: 10.1086/170411

Wright, E. L., Eisenhardt, P. R. M., Mainzer, A. K., et al. 2010, AJ, 140, 1868, doi: 10.1088/0004-6256/140/6/1868

Yanny, B., Rockosi, C., Newberg, H. J., et al. 2009, AJ, 137, 4377, doi: 10.1088/0004-6256/137/5/4377

Zinn, R., \& West, M. J. 1984, ApJS, 55, 45, doi: 10.1086/190947

Zoccali, M., Valenti, E., \& Gonzalez, O. A. 2018, A\&A, 618, A147, doi: 10.1051/0004-6361/201833147

Zolotov, A., Willman, B., Brooks, A. M., et al. 2009, ApJ, 702, 1058, doi: 10.1088/0004-637X/702/2/1058

Zorotovic, M., Catelan, M., Smith, H. A., et al. 2010, AJ, 139, 357, doi: 10.1088/0004-6256/139/2/357 